\title{
5 Die geschlossene Venerologische Station in Leipzig-Thonberg
}

\subsection{Das Fürsorgeheim Leipzig-Thonberg und das Stadtkrankenhaus Thonberg}

Die geschlossene Venerologische Station in Leipzig-Thonberg ging auf Beschluss der Landesverwaltung Sachsen direkt aus dem Fürsorgeheim LeipzigThonberg hervor. Das 1946 eingerichtete Fürsorgeheim für Geschlechtskranke Leipzig-Thonberg war der Vorläufer der geschlossenen Venerologischen Station. Mit dem Wechsel der Trägerschaft im Jahr 1952 vom Land Sachsen zur Stadt Leipzig gingen die Liegenschaft, die Einrichtungsgegenstände und das Personal des Fürsorgeheims direkt in die geschlossene Venerologische Station des Stadtkrankenhauses Thonberg über. Dieser nahtlose Übergang verdeutlicht die Kontinuität zwischen Fürsorgeheimen für Geschlechtskranke und den geschlossenen Venerologischen Stationen. Nicht einmal der rechtliche Rahmen hatte sich geändert. Sowohl für das Fürsorgeheim als auch für die geschlossene Venerologische Station des Stadtkrankenhauses Thonberg in Leipzig-Thonberg galt der SMAD-Befehl Nr. 273.

Nach dem Abzug der US-amerikanischen Alliierten marschierten die sowjetischen Truppen in Leipzig ein und trafen mit dem SMAD-Befehl Nr. 25 (Kap. 3.2) Vorkehrungen für die stationäre Zwangsbehandlung von syphiliskranken Personen in Leipzig. Für diesen Zweck wurden seit November 1945 unter anderem in der Klinik für Haut- und Geschlechtskrankheiten des Stadtkrankenhauses 
St. Jakob über 100 Betten zur Verfügung gestellt und Hospitalisierungen durchgesetzt. ${ }^{397}$ Doch wie in anderen Städten kam es auch in Leipzig zu Problemen mit der Umsetzung des SMAD-Befehls Nr. 25. Nicht zuletzt vor diesem Hintergrund trat im Februar 1946 der SMAD-Befehl Nr. 30 in Kraft. In der Folge wurden in Leipzig acht Ambulatorien und neun Sanierungsstellen zur Versorgung von Geschlechtskrankheiten eingerichtet. Zusätzlich wurden 1.430 Betten in Krankenhäusern für die Unterbringung und Behandlung von Geschlechtskranken bereitgestellt. ${ }^{398}$ Mit Wiedereröffnung der Universität Leipzig am 5. Februar 1946 konnten im Frühjahr desselben Jahres die sogenannten Pflichtkurse zur Aus- und Weiterbildung von Venerologen an der Hautklinik der Universität Leipzig aufgenommen und in regelmäßigen Abständen durchgeführt werden . Darüber hinaus konnte die Landesverwaltung Sachsen, Abteilung Gesundheitswesen, in einem Schreiben vom 28. März 1946 der SMAD mitteilen, „daß in der städtischen Arbeitsanstalt, die schon nach dem 1. Weltkrieg zur Unterbringung Geschlechtskranker gedient hatte, ein Fürsorgeheim mit 8o Betten zur zwangsweisen Behandlung von Prostituierten und von Personen, die sich der Behandlung entzogen hatten, eingerichtet worden war“. 399

Die „Zwangsarbeitsanstalt zu St. Georg in Leipzig-Thonberg“ war am 8. November 1892 auf dem Grundstück Riebeckstraße 63 eingerichtet worden. Sie war als Arbeitshaus sowie als ein Versorgten- und Siechenhaus geplant. Auf dem Grundstück befanden sich um die Jahrhundertwende Verwaltungsgebäude, ein Wirtschaftsgebäude, zwei Männerhäuser, eine Abteilung für Jugendliche, ein Frauenhaus und ein Pförtnerhaus mit Polizeiwache. ${ }^{400} 1909$ wurde die „Zwangsarbeitsanstalt zu St. Georg in Leipzig-Thonberg“ in die „Städtische Arbeitsanstalt“ überführt. Ab 1923 unterstand sie dem Wohlfahrtsamt. Die Gebäude in der Riebeckstraße 63 wurden während des Zweiten Weltkriegs vom Arbeitsamt genutzt, um hier unter anderem Fremd- und Zwangsarbeiter aus Polen zu registrieren. Obwohl im März 1946 in der ersten Etage des Fürsorgeheims Leipzig-Thonberg 8o Betten für geschlechtskranke Frauen eingerichtet waren, blieb die Stadt Leipzig weit hinter den Erwartungen der SMAD zurück. Vorgesehen waren 250 Betten für die Zwangshospitalisierung von geschlechtskranken Frauen. ${ }^{401}$ Doch bauliche Probleme in der zweiten Etage sowie die Belegung des Erdgeschosses mit Zwangsarbeitern aus Polen verhinderten die Erhöhung der Bettenzahl auf 250. Im Juli 1946 gelang es, 128 Betten im Fürsorgeheim für geschlechtskranke Frauen einzurichten. ${ }^{402}$ Weiterhin sollten

397 Schreier: Die Entwicklung der Dermato-Venerologie (Anm. 95), S. 101.

398 StAL: StVuR Nr. 14744. Hauptgesundheitsamt Leipzig. Leipzig, den 12. Juni 1946, Bl. 24.

399 Schreier: Die Entwicklung der Dermato-Venerologie (Anm. 95), S. 103.

400 0. A. (1892) Das neue Arbeitshaus zu St. Georg in Leipzig-Thonberg" In: Vereinigung Leipziger Architekten und Ingenieure (Hrsg.) Leipzig und seine Bauten. 285-290. Gebhardt Leipzig.

401 StAL: StVuR Nr. 7093. Landesverwaltung Sachsen. Gesundheitswesen an den Oberbürgermeister der Stadt Leipzig. Dresden, den 1. Juli 1946, Bl. 1.

402 StAL: StVuR Nr. 7093. Stadtgesundheitsamt. An die Landesverwaltung, Abteilung: Gesundheitswesen. Leipzig, den 18. Juli 1946, Bl. 3. 
die Räumung des Erdgeschosses und die Instandsetzung der zweiten Etage mit Nachdruck vorangetrieben werden.

Ursprünglich war auch die Unterbringung von geschlechtskranken Männern im Fürsorgeheim Leipzig-Thonberg geplant. Doch ab Sommer 1946 wurde in Leipzig-Dösen eine Einrichtung für geschlechtskranke Männer eröffnet, die vorerst aus „einem grossen Zimmer mit 13 Betten“ bestand. Im Bedarfsfall konnte die ganze Etage für die „zwangsbehandelten Männer in Anspruch genommen werden“. ${ }^{403}$ Aus verschiedenen Sitzungsprotokollen des „Beirats zur Bekämpfung von Geschlechtskrankheiten“ geht hervor, dass auf die HwG-Listen nur wenige Männer aufgenommen wurden und somit das Fürsorgeheim für männliche Patienten in Leipzig-Dösen kaum belegt war. ${ }^{404}$ Aus der Zeit zwischen 1946 und 1948 sind diverse Schreiben überliefert, in denen über den weiteren Ausbau der zweiten Etage des Fürsorgeheims Leipzig-Thonberg verhandelt wurde, um den Erwartungen der SMAD gerecht zu werden. Deutlich wird, dass in dem Fürsorgeheim anfänglich 120 bis 130 Zwangseingewiesene versorgt wurden. Seit 1948 waren noch durchschnittlich 50 bis 60 Zwangseingewiesene im Fürsorgeheim untergebracht. ${ }^{405}$ Aufgrund der geringeren Patientenzahlen war seit 1948 von einem Ausbau des Fürsorgeheims keine Rede mehr. Vielmehr findet sich in den Akten aus jener Zeit der Vorschlag, das Fürsorgeheim Leipzig-Thonberg vollständig zu schließen, zumindest aber zu verlegen. So heißt es unter anderem in einem Schreiben vom 30. September 1950: „Das Fürsorgeheim Thonberg war im Sommer ds. Js. mit seinen Patienten auf 24 zusammengesunken. Wir hatten deshalb gebeten, darauf hinzuarbeiten, daß eine Verlegung dieser wenigen Patienten nach einem anderen Krankenhaus vorgenommen würde. " ${ }^{006}$ Der Gedanke, das Fürsorgeheim Leipzig-Thonberg zu schließen, war eingebettet in die Planung einer Zwangsbehandlungsstätte für asoziale geschlechtskranke Frauen im Landesmaßstab. In diesem Zusammenhang wurden Städte wie Grimma aber auch Colditz genannt, in denen die Landesbehandlungsstelle eröffnet werden sollte. Der Vorschlag, das Fürsorgeheim Leipzig-Thonberg aufzulösen bzw. in eine Landesfürsorgeanstalt zu überführen, war auch vor dem Hintergrund entstanden, dass bis zu 8o Prozent der Zwangseingewiesenen nicht aus der Stadt Leipzig, sondern aus anderen Landkreisen kamen. ${ }^{407}$

Schließlich führte das Ministerium für Gesundheitswesen des Landes Sachsen eine Entscheidung über die Zukunft des Fürsorgeheims Leipzig-Thonberg her-

403 StAL: StVuR Nr. 7093. Stadtgesundheitsamt. An die Landesverwaltung (Anm. 402).

404 Vgl. StAL: StVuR Nr. 7345. Sitzungsprotokolle des Beirats zur Bekämpfung von Geschlechtskrankheiten von 1946-1948.

405 StAL: StVuR Nr. 14744. Sozialamt. An das Stadtgesundheitsamt. Betr. Fürsorgeheim Thonberg. Leipzig, den 31. Dezember 1948. Bl. 210.

406 StAL: StVuR Nr. 14739. Heim für soziale Betreuung, früher Städt. Arb. Anstalt Leipzig, Riebeckstr. 63. An das Sozialamt. Leipzig, der 30. September 1950, Bl. 9.

407 StAL: StVuR Nr. 14739. Amtsleitung Gesundheitsamt. An das Ministerium für Arbeit und Gesundheitswesen des Landes Sachsen, Leipzig, den 5. März 1950, Bl. 27. 
bei, wie einem Aktenvermerk vom 31. August $1951 \mathrm{zu}$ entnehmen ist. Darin wurde festgelegt, dass „das Fürsorgeheim Thonberg haushalts- und verwaltungsmäßig 1952 vom Stadtgesundheitsamt Leipzig übernommen werden muß ${ }^{\prime 4}{ }^{408}$ Mit dieser Entscheidung vonseiten der Landesregierung endete formal die Existenz des Fürsorgeheims für Geschlechtskranke Leipzig-Thonberg. Mit der Übernahme der Einrichtung durch die Stadt Leipzig wurde auch die Bezeichnung „Fürsorgeheim“ schrittweise gestrichen. Wurde sie noch im Januar 1952 als „Städtisches Krankenhaus Fürsorgeheim Thonberg“ bezeichnet, so nennt der Bezirksbeauftragte der Landesregierung Sachsen in einem Schreiben an das Dezernat Gesundheitswesen Leipzig im Februar 1952 die Einrichtung schlicht „Stadtkrankenhaus Thonberg“. ${ }^{409}$

Kurz nach der Übernahme des Fürsorgeheims Leipzig-Thonberg durch die Stadt Leipzig und die Umbenennung des Fürsorgeheims in „Stadtkrankenhaus Thonberg" im Jahr 1952 erschien in der Sächsischen Zeitung ein Artikel über das Stadtkrankenhaus Thonberg. Darin heißt es: „In diesem ,Führungshaus wird heute noch Naziliteratur geführt. Auch eine Hitlerbüste ist noch vorrätig (oder war es zur Zeit der Überprüfung). In den Krankenräumen stehen alte Wehrmachtbettstellen mit allem alten Zubehör, darunter z.B. das ,Hoheits'Abzeichen der abgrundtief verkommenen Verbrecherbande, die Deutschland bis zu sieben Jahre versklavte. (...) Nichts ist in den Räumen außer den Kasernenbetten, keine Vorhänge, kein Schmuck, keine Bilder, keine Inschriften, keine Blumen - alles ist kalt und lieblos und kulturlos. “410 Dieser Darstellung widersprach die Bezirks- und Kreisbeauftragte zur Bekämpfung der Geschlechtskrankheiten, Frau Dr. med. Bittner-Pietsch, in einer Sitzung des Dezernats Gesundheitswesen kategorisch. Zwar gäbe es die Hakenkreuzinsignien in Form von Stempeln auf den Bettgestellen, doch weder eine Hitlerbüste noch NS-Literatur wären im Krankenhaus Leipzig-Thonberg vorhanden. Die in der Sächsischen Zeitung „monierte einfache Einrichtung entspricht den lt. Befehl zo erlassenen Ausführungsbestimmungen“, so Bittner-Pietsch. Weiter führte sie aus, dass bauliche „Veränderungen und Renovierungen nicht vorgenommen [wurden], da seit langem eine Ungewissheit über den weiteren Verbleib dieses Heimes im dortigen Gebäude bestand“."411

Im Mai desselben Jahres, so geht aus einem Bericht über die Kontrollbesichtigung des städtischen Krankenhauses hervor, wurden Renovierungsarbeiten in der geschlossenen Venerologischen Station des Stadtkrankenhauses Thonberg durchgeführt. Die Einrichtung „wird grundlegend renoviert, und zwar sind neue Doppelfenster eingesetzt worden, und die Räume werden neu ge-

408 StAL: StVuR Nr. 14739. Ministerium für Gesundheitswesen des Landes Sachsen. Aktenvermerk. Dresden, den 3. August 1951. Bl. 2.

409 StAL: StVuR Nr. 14739. Bezirksbeauftragte der Landesregierung Sachsen. An das Dezernat Gesundheitswesen. Leipzig, den 15. Februar 1952, Bl. 25.

410 StAL: StVuR Nr. 7093. Abschrift. Sächsische Zeitung vom 15. Februar 1952, 7. Jhrg. Nr. 39, Bl. 30.

411 StAL: StVuR Nr. 7093. Dezernat Gesundheitswesen. Bericht. Leipzig, den 29. Februar 1952, Bl. 29. 
malt. Zu wünschen wäre, daß in den beiden Sälen die dort stehenden eisernen Öfen entfernt und noch bevor die Renovierung beendet ist, durch größere Berliner Öfen ersetzt werden. Die eisernen Öfen reichen nicht aus, im Winter den gesamten Saal durchzuheizen. (...) Der Fußboden wird ausgebessert, jedoch wäre es wünschenswert, hier nach Möglichkeit Steinholzfußboden auszulegen. (...) Der Baderaum und die Waschanlage sind gekachelt worden (...), der Behandlungsraum wird ebenfalls gekachelt." " ${ }^{122}$ Die Beschreibung der Räume durch die Sächsische Zeitung aber auch die Auflistung der Renovierungsarbeiten machen noch einmal deutlich, dass sich am Zustand und der Ausstattung der Räume seit Gründung des Fürsorgeheims im Jahr 1946 kaum etwas geändert hatte.

Nicht nur der Zeitungsartikel hatte Anfang des Jahres 1952 für Unruhe gesorgt. $\mathrm{Zu}$ dieser Zeit häuften sich auch Berichte über undiszipliniertes Verhalten unter den Zwangseingewiesenen. In einem Brief des leitenden Arztes Dr. med. Hoffmann, der erst vor wenigen Wochen die Leitung des Krankenhauses übernommen hatte, heißt es: „Die Disziplinlosigkeit der bei uns in geschlossener Anstalt zur Zwangsbehandlung untergebrachten Patientinnen (...) nimmt in letzter Zeit in der Krankenanstalt in erschreckendem Maße zu. Es scheint so, als ob die in letzter Zeit gehäuften Kontrollbesuche der verschiedensten Institutionen bei den Patientinnen die Meinung hervorgerufen hat, als seien sie unschuldige Opfer, die zu Unrecht festgehalten werden. Zugleich ist ihnen bekannt, daß keine Möglichkeit einer Bestrafung besteht. Dies führt zu täglichen Konflikten, sei es, daß nachts geraucht wird und die anderen Patientinnen dadurch gestört werden, sei es durch blutige Schlägereien untereinander oder sei es durch persönliche Beleidigung der Oberschwester und böswilliger Kritik der ärztlichen Anordnungen und dergleichen mehr. "Nach dieser Schilderung bat der leitende Arzt um präzise Angaben, „was zu tun ist, bei Behandlungsverweigerung, bei Beleidigung des Arztes, bei Schlägereien oder etwa tätlicher Bedrohung der Oberschwester oder des Personals“. ${ }^{413}$

In Reaktion auf diese Beschreibung schlug Bittner-Pietsch die Einführung härterer Straf- und Sanktionsmöglichkeiten vor. So könne „für besonders disziplinlose Patientinnen eine Besuchs- bezw. Paketsperre eintreten. Dies wurde früher so gehandhabt. “ Darüber hinaus sei „es notwendig, ein Einzelzimmer parat zu halten, in dem Patientinnen, die die anderen Patientinnen durch ihr renitentes Verhalten aufputschen, untergebracht werden können. "Eventuell müssten ,auch noch andere Strafen ausgesetzt werden, die evtl. in einer Sicherungsverwahrung münden können. " ${ }^{114}$ Doch diesen Vorschlägen wurde

412 StAL: StVuR Nr. 7093. Bericht über die Kontrollbesichtigung des städtischen Krankenhaus - Fürsorgeheim Thonberg. Leipzig, den 4. Mai 1952, Bl. 36.

413 StAL: StVuR Nr. 7093. Städt. Krankenhaus Fürsorgeheim Thonberg. An die Bezirks- und Kreisbeauftragte. Leipzig, den 14. Februar 1953, Bl. 24.

414 StAL: StVuR Nr. 7093. Bezirksbeauftragte. An das Dezernat Gesundheitswesen. Leipzig, den 16. Februar 1952, Bl. 25. 
vorerst vonseiten des Dezernats Gesundheitswesen eine Absage erteilt. Vielmehr müsse eine Grundlage, eine Ordnung oder Hausordnung erstellt werden, in der für alle verbindlich geregelt sei, welche Verhaltensformen im Stadtkrankenhaus Thonberg sanktioniert werden und wie entsprechende Sanktionen aussehen könnten. Gleichzeitig fragte das Dezernat beim Ministerium für Gesundheitswesen des Landes Sachsen an, ob eine solche Hausordnung für Sachsen vorliege. In einem Antwortschreiben des sächsischen Gesundheitsministeriums hieß es dazu: „Wir teilen dazu mit, daß Vorschläge für eine einheitliche Hausordnung in stationären Einrichtungen vor mehreren Monaten nach Berlin gegeben worden sind, jedoch leider von dort eine Bestätigung bisher noch nicht erfolgt ist. Es besteht daher die Absicht, für Sachsen in kürzester Zeit eine solche herauszugeben. "415 Im selben Schreiben wird die Abteilung Gesundheitswesen gebeten, einen Entwurf für eine Hausordnung zu erstellen.

\subsection{Das Krankenhaus Leipzig-Thonberg und die rechtswidrigen Einweisungen in die geschlossene Venerologische Station}

Seit in Kraft treten der „Verordnung zur Verhütung und Bekämpfung von Geschlechtskrankheiten“ im Jahr 1961 war vor allem der $\mathbb{2}$ dafür missbraucht worden, Mädchen und Frauen unter dem Verdacht häufig wechselnder Geschlechtspartner sofort in eine geschlossene Venerologische Station einzuweisen. ${ }^{416}$ Gerade der $\mathbb{2} 22$ bot den zuführenden Polizisten aber auch den aufnehmenden Venerologen viel Interpretationsspielraum. Diese Einweisungspraxis war nach Auffassung der Staatsanwaltschaft Leipzig rechtswidrig, wie Ermittlungsakten zeigen. Infolge ihrer Ermittlungen sollte die geschlossene Venerologische Station des Stadtkrankenhauses Thonberg aufgelöst, zumindest aber umstrukturiert werden.

Die Praxis der rechtswidrigen Einweisungen lässt sich an den Schilderungen einer Zeitzeugin nachvollziehen: „Naja, die haben mich da, die haben uns, das erste Mal haben die uns, die haben uns weggefangen auf der Straße. Wir waren im Kino wohl gewesen. Dann haben die eine Ausweiskontrolle gemacht und rein ins Auto. Und auf das Nachtambulatorium, Kohlgartenstraße." Auch bei den weiteren Einweisungen im Jahr 1961 und 1963 wurde die Zeitzeugin jedes Mal von der Polizei aufgegriffen. Nach einer Untersuchung in der Kohlgartenstraße wurde die Zeitzeugin von der Polizei direkt in die geschlossene Venerologische Station des Stadtkrankenhauses Thonberg gebracht. Dort wurde sie ein weiteres Mal untersucht. „Ja, ich weiß nur, dass die immer mit so einem Rohr untersucht haben, mit so Glasrohren und dann mit so einem Stab da rein, so einem heißen oder was das war. (...) Und man gab uns Spritzen,

415 StAL: StVuR Nr. 7093. Ministerium für Gesundheitswesen des Landes Sachsen. An den Rat der Stadt Leipzig Gesundheitswesen. Dresden, den 24. Mai 1952, Bl. 38.

416 Verordnung zur Verhütung und Bekämpfung von Geschlechtskrankheiten (Anm. 23), S. 88. 
ich weiß nicht wie viel, so und da hieß es, da muss jeden Tag untersucht werden." Der Alltag auf der geschlossenen Venerologischen Station war bereits Anfang der 196oer Jahre klar geregelt, wie die Aussagen der ehemaligen Zwangseingewiesenen bestätigen: „Na, früh die Untersuchung und dann gab es Frühstück. Und dann waren, ja, ich glaube, das waren dann Gartenarbeiten oder überhaupt so irgendwo was, mussten wir uns beschäftigen. Wir kamen ja nicht raus. “417 Vor allem die Beschäftigung im Außengelände, die Gartenarbeiten, waren eine Tätigkeit, die auch für die 1940er und 1950er Jahre in anderen Quellen überliefert wird. ${ }^{418}$ Nach der Arbeit gab es Freizeit und um 22 Uhr war Nachtruhe. „Da wurde das Licht ausgemacht. Und dann wurde zugeschlossen, alles wieder, nochmal kontrolliert und dann war Ruhe. Also (...) Radio und solche Sachen, das gab es ja gar nicht." Die Dauer ihres ersten Aufenthalts betrug 10 Wochen und entsprach nicht den medizinischen Standards der Zeit. Bei der Entlassung erhielt die Frau „keine Entlassungspapiere, wir kriegten gar nichts“."${ }^{419}$

Ihre Aussagen zu den Zwangseinweisungen in den Jahren 1960, 1961 und 1963 in die geschlossene Venerologische Station weisen Parallelen zur rechtswidrigen Einweisung einer jungen Frau mit den Initialen D.H. im Jahr 1963 auf. Diese hatte im Dezember 1963 und Februar 1964 jeweils einen Diebstahl begangen. In diesem Zusammenhang war die Staatsanwaltschaft auf D.H. und ihre Lebenssituation aufmerksam geworden. So geht aus den Akten hervor, dass sie am 26. Februar 1964 in ein Ambulatorium in der Kohlgartenstraße gebracht wurde, „weil sie sich herumgetrieben und wahllos Männerbekanntschaften angeknüpft hatte. Wegen Verdachts der Geschlechtskrankheit erfolgte Einweisung in das Krankenhaus Thonberg. Hier war die Jugendliche vom 26. 2. bis 3. 4. 1964 untergebracht. Der Krankheitsverdacht bestätigte sich nicht. Anschließend erfolgte auf dem Verwaltungsweg Einweisung in einen Jugendwerkhof." “220

Zwei Punkte alarmierten die Staatsanwaltschaft, vertreten durch Herrn Dr. jur. Fräbel. Zum einen war es die Zwangseinweisung auf eine geschlossene Station. Zum anderen war es die Dauer der Zwangseinweisung. Vor dem Hintergrund der 1961 erlassenen „Verordnung zur Verhütung und Bekämpfung von Geschlechtskrankheiten “ untersuchte die Staatsanwaltschaft die Einweisung von D.H. Hierzu besuchte der Staatsanwalt das Stadtkrankenhaus Thonberg, die geschlossene Station für Geschlechtskranke, und prüfte vor Ort die Praxis der Einweisung. Im Anschluss an diese Besichtigung wurden erste Sofortmaßnahmen beschlossen. So schreibt Fräbel an den Rat der Stadt Leipzig:

417 Interview mit Frau LPB, geführt von Maximilian Schochow, Halle (Saale), den 21. April 2015.

418 StAL: StVuR Nr. 7345. Die Kreisbeauftragte zur Bekämpfung der Geschlechtskrankheiten. Protokoll über die Sitzung des Beirats zur Bekämpfung der Geschlechtskrankheiten am 16. August 1947. Leipzig, den 16. August 1947, Bl. 21.

419 Interview mit Frau LPB (Anm. 417).

420 StAL: StVuR Nr. 7121. Stadtstaatsanwalt. An den Rat der Stadt Leipzig. Leipzig, den 22. Mai 1964, Bl. 298. 
„Gegenwärtig gibt es in Leipzig als Krankenhaus zur Unterbringung und Behandlung von Geschlechtskranken nur das Städtische Krankenhaus Riebeckstrasse 63. Diese Anstalt ist als ,geschlossene“ Anstalt im Sinne der Gekra-VO [Geschlechtskrankheitsverordnung] anzusprechen. Nach den gesetzlichen Bestimmungen darf jedoch von der Einweisung in eine geschlossene Abteilung für Geschlechtskranke durch den Rat des Kreises - Abteilung Gesundheits- und Sozialwesen - erst dann Gebrauch gemacht werden, wenn anderen Massnahmen der Untersuchung und Behandlung nicht nachgekommen wird. ( $\$ 20$ Abs. 3 und $\mathbb{2} 22 \mathrm{Abs}$. 3 Gekra-VO). Es muss offen ausgesprochen werden, dass die Einweisung eines Kranken oder Krankheitsverdächtigen zur stationären Behandlung durch Ärzte der Ambulatorien nur in nichtgeschlossenen Krankenhäusern erfolgen darf. ( $\$ 19$ Gekra-VO)“, und weiter heißt es: „Ich muss als Staatsanwalt im Rahmen der Gesetzlichkeitsaufsicht darauf hinweisen, dass der ungesetzliche Zustand der generellen Einweisung von Kranken und Krankheitsverdächtigen in eine geschlossene Anstalt nicht zulässig ist.“"21

Die Analyse und die Forderung Fräbels waren klar. Die jahrelange Praxis der direkten Einweisung von geschlechtskranken krankheitsverdächtigen Frauen und Mädchen in die geschlossene Station war rechtswidrig. Daraus leitete er die Forderung ab, dass es künftig keine weiteren direkten Zwangseinweisungen in die geschlossene Venerologische Station von Leipzig geben dürfte. Vielmehr müssten erst einmal alle Geschlechtskranken und Krankheitsverdächtigen in einer Ambulanz, zumindest aber auf einer offenen Station medizinisch versorgt werden, wie es im $\mathbb{1} 19$ der „Verordnung zur Verhütung und Bekämpfung von Geschlechtskrankheiten" aus dem Jahr 1961 vorgesehen war.

Des Weiteren beschäftigte den Staatsanwalt die Dauer des Aufenthalts. Um diese Frage zu lösen, schlug er die Einsetzung einer Kommission vor, die sich mit folgenden vier Aspekten beschäftigen sollte: „I. Wie kann gewährleistet werden, dass in der offenen und in der geschlossenen Anstalt das Prinzip der Differenzierung nach Alter der Eingewiesenen, nach Rückfälligkeit und nach anderen pädagogischen und medizinischen Gesichtspunkten erfolgt. II. Welche Möglichkeiten gibt es, die Dauer des Krankhausaufenthalts auf ein Mindestmass des medizinisch Erforderlichen herabzusetzen. III. Welche Möglichkeiten der sinnvollen Beschäftigung der Eingewiesenen gibt es (...). IV. Welche Sofortmassnahmen müssen eingeleitet werden, um in der Anstalt selbst alles auszuschalten, was der Würde des Menschen nicht entspricht (z.B. Blechnäpfe, verdreckte Matratzen, ausreichende Anstaltskleidung usw.). " ${ }^{222}$ Vor allem der letzte Punkt macht deutlich, dass noch Gegenstände des alten Fürsorgeheims Thonberg bis Mitte der 196oer Jahre in der geschlossenen Venerologischen Station genutzt wurden - unter anderem die Blechnäpfe oder mit Stroh gefüllte unsaubere Matratzen.

421 StAL: StVuR Nr. 7121. Stadtstaatsanwalt (Anm. 420), Bl. $299 f$. 422 StAL: StVuR Nr. 7121. Stadtstaatsanwalt (Anm. 420), Bl. 300. 
Die staatsanwaltlichen Ermittlungen hatten unter den verantwortlichen Venerologen für Unruhe gesorgt. Der Dermatologe der Poliklinik Kohlgartenstraße und Kreisdermatovenerologe Dr. med. Sieler, der D.H. in das Krankenhaus Leipzig-Thonberg zwangseingewiesen hatte, beschwerte sich bei Kreisarzt OMR Dr. med. Riemschneider: „Wir haben immer wieder Schwierigkeiten in der Zusammenarbeit mit der Staatsanwaltschaft bei Einweisung von weiblichen Personen in das Krankenhaus Thonberg, sofern diese lediglich zugeführt sind, aber nicht als Person mit hwG aufgesetzt sind. Die Staatsanwaltschaft hat uns mehr oder minder verblümt in diesen Fällen den Vorwurf der Freiheitsberaubung gemacht. " ${ }^{423}$ Die verantwortlichen Venerologen in Leipzig wussten demnach um die gesetzeswidrigen Einweisungen. Dennoch hatten sie seit Jahren daran festgehalten und verdächtige Frauen in die geschlossene Station zwangseingewiesen.

In seinem Schreiben schlug der Kreisdermatovenerologe Sieler gleichzeitig eine Lösung vor, wie das Problem der generell geschlossenen Venerologischen Station zu lösen wäre: „Nach wie vor soll das 2. Stockwerk im Krankenhaus Thonberg als geschlossene Station geführt werden. (...) Die untere Station soll als offene Station geführt werden, wobei z.B. zugeführte Personen ohne hwG oder sonstige weibliche säumige Personen aufgenommen werden können. Wir würden dadurch für uns eine ganz erheblich bessere Rechtssituation erreichen. "In der Vorstellung von Sieler sollte die Einweisung von kranken sowie krankheitsverdächtigen Mädchen und Frauen künftig wie folgt organisiert werden: Die nach Thonberg eingewiesenen Patientinnen werden „auf der offenen Station Krankenhausbekleidung empfangen, die Privatkleidung wird unter Verschluss genommen, so dass diese Personen gleichfalls nicht entweichen können. Aus disziplinarischen Gründen wäre allerdings Besuch von aussen auf dieser Station nicht angebracht. “"24 Diejenigen Personen, die dann die Bedingungen für eine geschlossene Unterbringung nach der „Verordnung zur Verhütung und Bekämpfung von Geschlechtskrankheiten“ erfüllen, sollten nach der Einweisung in die offene Station sofort in die geschlossene Station in die zweite Etage gebracht werden.

In der Folge fanden zahlreiche Besichtigungen des Stadtkrankenhauses Thonberg und Besprechungen über die Umgestaltung der geschlossenen Venerologischen Station statt. ${ }^{425}$ Stets wurde bei diesen Besichtigungen reklamiert, dass das Dach abgedichtet und Heizungsmöglichkeiten geschaffen werden müssten, um die zweite Etage als geschlossene Station betreiben zu können. Erst diese Umbauarbeiten würden die Trennung in eine offene Station in der

423 StAL: StVuR Nr. 7121. Poliklinik Kohlgartenstraße. An den Rat der Stadt. Herrn Kreisarzt OMR. Dr. med. Riemschneider. Leipzig, den 29. April 1964, Bl. 296.

424 StAL: StVuR Nr. 7121. Poliklinik Kohlgartenstraße (Anm. 423).

425 Vgl. StAL: StVuR Nr. 7121. Rat der Stadt Leipzig. Niederschrift. Am 22. August 1964 fand eine Aussprache im Krankenhaus Thonberg statt - Riebeckstrasse mit anschließender Begehung. Leipzig, den 3. September 1964, Bl. 277. 
ersten Etage für auffällige Patientinnen und eine geschlossene Venerologische Station für HwG-Frauen in der zweiten Etage ermöglichen. Zusätzlich sollte auf beiden Stationen eine Differenzierung bei der Belegung der Einzelräume erfolgen, sodass „,vermieden wird, dass unerfahrene Jugendliche von älteren Personen negativ beeinflusst werden“. Um den Anforderungen der Staatsanwaltschaft Rechnung tragen zu können, sollte der „bisher veranschlagte Pauschalaufenthalt im Krankenhaus Thonberg von 4 Wochen“ künftig an den medizinischen Notwendigkeiten der individuellen Patientin orientiert werden. Schließlich wäre es erforderlich, eine Hausordnung zu erstellen, um disziplinarische Schwierigkeiten innerhalb der Station in den Griff zu bekommen, wie aus einem Protokoll hervorgeht. ${ }^{426}$

Zwar wurde im September 1964 ein Minimalplan zur Umgestaltung des Krankenhauses Thonberg erstellt, der die Reparatur des Daches vorsah, die Instandsetzung der Öfen und die Zweiteilung der Station in eine offene Station mit 17 Betten und eine geschlossene Station mit 46 Betten. ${ }^{227}$ Doch die Teilung der Station in eine offene und eine geschlossene Station wurde weder 1964 noch 1965 umgesetzt. Stattdessen wurde das Stadtkrankenhaus Thonberg am 1. Juli 1964 durch die Poliklinik Kohlgartenstraße übernommen. Infolge des Trägerwechsels wurde lediglich der Name des Stadtkrankenhauses geändert. Um zu einer einheitlichen Bezeichnung zu kommen, wurde festgelegt: „Poliklinik Kohlgartenstraße, Krankenstation Thonberg, 705 Leipzig 5, Riebeckstr. 63." ${ }^{228}$ Damit gehörte die geschlossene Venerologische Station seit 1964 verwaltungstechnisch zur Poliklinik Kohlgartenstraße und trug seit 1965 den Namen „Krankenstation Thonberg“.

An der rechtswidrigen Einweisungspraxis wurde auch unter dem neuen Träger, der Poliklinik Kohlgartenstraße, festgehalten. Geschlechtskranke und krankheitsverdächtige Mädchen und Frauen wurden, wie in den Jahren zuvor, unmittelbar in die geschlossene Venerologische Station des „Krankenhauses Thonberg " zwangseingewiesen. Eine Trennung der Station in eine geschlossene und eine offene hätte vielen zwangseingewiesenen Mädchen und Frauen die geschlossene Station erspart. Sie wären ambulant oder auf einer offenen Station versorgt worden. Eine Teilung hätte auch die Einweisung rechtskonform gestaltet. Doch auch nach der Übernahme der geschlossenen Venerologischen Station durch die Poliklinik Kohlgartenstraße fanden Verstöße gegen geltendes Recht statt, da keine zwei Stationen geschaffen wurden. Möglicherweise waren solche Gesetzeswidrigkeiten nicht nur in Leipzig-Thonberg Routine, sondern eine Praxis, die auch in anderen geschlossenen Venerologischen Stationen der DDR angewandt wurde.

426 StAL: StVuR Nr. 7121. Protokoll über die beiden Begehungen im Krankenhaus Thonberg. Leipzig, den 22. Mai 1964, Bl. 289-293, hier: Bl. 290.

427 StAL: StVuR Nr. 7121. Poliklinik Kohlgartenstraße. An den Kreisarzt. Betr. Städt. Krankenhaus Thonberg. Minimalplan 1964. Leipzig, den 16. September 1964, Bl. 264.

428 StAL: StVuR Nr. 7121. Poliklinik Kohlgartenstraße. An den Rat der Stadt Leipzig. Leipzig, den 2. Februar 1965, BI. 262. 


\subsection{Die Hausordnung der Krankenstation Thonberg}

Eine räumliche Teilung der Krankenstation Thonberg in eine offene und eine geschlossene Venerologische Station war auch 1966 nicht realisiert worden. Weiterhin wurde mit jeder Einweisung von Mädchen und Frauen, die nicht auf einer HwG-Liste standen, gegen bestehendes Recht der DDR verstoßen. Mit einer Hausordnung für die Krankenstation sollte diesen Verstößen zumindest auf dem Papier entgegengewirkt werden. In den ersten Monaten des Jahres 1966 war eine Hausordnung entworfen und mehrfach überarbeitet worden, die Sieler am 5. Mai 1966 an den Rat der Stadt Leipzig, Abteilung Gesundheitswesen sandte: „In der Anlage erhalten Sie die endgültig ausgearbeitete Hausordnung, die in der vorliegenden Form nunmehr Gültigkeit erlangen soll. Nachdem ich dankenswerter Weise von interessierten Vertretern der Staatsmacht und der Abt[eilung] Gesundheits- und Sozialwesen kollegiale Hinweise zur Abfassung dieser Hausordnung erhalten habe, dürfte einer Inkraftsetzung der Hausordnung in der jetzigen Form nichts mehr im Wege stehen. Bei der jetzigen Form haben wir vor allem auf die rechtlichen Grundlagen der Einweisung in die Krankenstation Thonberg zurückgegriffen, andererseits haben wir auch eine entsprechende Rechtsmittelbelehrung mit eingebaut. Weiterhin haben wir die erzieherischen Momente in der Hausordnung in den Vordergrund gestellt, so z.B. vornehmlich die Verantwortung des Kollektivs bei der Einhaltung der Ordnung und die Möglichkeit der Arbeit für die Patienten. “429

Obwohl Sieler in seinem Anschreiben erzieherische Momente hervorhob, erinnerte die „Hausordnung für die Krankenstation Thonberg“ “430 weder in ihrem Aufbau noch in den dort fixierten Vorschriften an die Hausordnung der geschlossenen Venerologischen Station der Poliklinik Mitte in Halle (Saale). So fehlten vor allem die erzieherischen Ziele: Das in der Hausordnung von Halle (Saale) fixierte Vorhaben, die Zwangseingewiesenen zu gesetzestreuen Bürgerinnen zu erziehen, war in der „Hausordnung für die Krankenstation Thonberg“ ebenso wenig niedergeschrieben wie die Formung von ,sozialistischen Persönlichkeiten“. Auch die ausführlichen Beschreibungen zur Aufnahme in die geschlossene Venerologische Station, zum Tagesablauf oder zu den sogenannten „Arbeitstherapien“, die in der Hausordnung von Halle (Saale) enthalten waren, sind in der „Hausordnung für die Krankenstation Thonberg“ nicht $z u$ finden.

Im Gegensatz zur Hausordnung von Halle (Saale) wurden in der Hausordnung für Leipzig-Thonberg die rechtlichen Aspekte stark herausgearbeitet. Gleich in den $\$ \$ 1$ und 2 wurden die rechtlichen Grundlagen der Krankenstation dargelegt. Der $\mathbb{1}$ ging auf die „Verordnung zur Verhütung und Bekämpfung der

429 StAL: StVuR Nr. 7121. An den Rat der Stadt Leipzig. Betr.: Hausordnung für die Krankenstation Thonberg. Leipzig, den 5. Mai 1966, Bl. 256.

430 StAL: StVuR Nr. 7121. Hausordnung für die Krankenstation Thonberg, 705 Leipzig, Riebeckstr. 63, Bl. 257-261. 
Geschlechtskrankheiten“ vom 23. Februar 1961 ein und im $\$ 2$ wurden Möglichkeiten zur Beschwerde ausgeführt. Die $\mathbb{S} 33$ und 4 sollten dazu dienen, die Funktion der Krankenstation Thonberg zu bestimmen. Hierzu heißt es im $\mathbb{s}$ : „Der Aufenthalt einer Patientin in der Krankenstation Thonberg dient in Verdachtsfällen zur Untersuchung auf Vorliegen von Geschlechtskrankheiten bzw. zur diagnostischen Feststellung derselben. “Und im $\$ 4$ heißt es: „Ist eine Geschlechtskrankheit bereits ausserhalb der Krankenstation festgestellt worden oder wird diese während des Aufenthalts einer Patientin in der Krankenstation Thonberg nachgewiesen, erfolgt die erforderliche Behandlung und eine entsprechende Nachkontrolle, die zum Nachweis der Ausheilung dient.“ Darüber hinaus war im $\mathbb{S} 5$ geregelt, dass „der Aufenthalt einer Patientin in der Krankenstation Thonberg auch zur Durchführung dieser Nachkontrollen dienen" konnte. ${ }^{431}$

Zur rechtlichen Absicherung der Einweisung von geschlechtskranken und

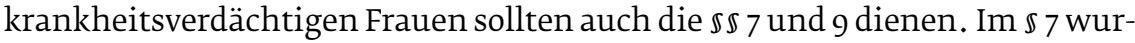
de die Zweiteilung der Einrichtung festgelegt: „In der Krankenstation Thonberg wird ein geschlossener und offener Teil unterschieden. In den geschlossenen Teil werden diejenigen Patientinnen eingewiesen, die gemäss der einschlägigen Verordnung und gemäss der Richtlinie zur Durchführung der Verordnung zur Verhütung und Bekämpfung der Geschlechtskrankheiten vom 12. Oktober 1965, erschienen in den Verfügungen und Mitteilungen des Ministeriums für Gesundheitswesen Nr. 21 vom 5. November 1965, als dringend krankheitsverdächtig gelten und sich auf Grund ihrer Verhaltensweise einer turnusgemässen Untersuchung (Kontrolluntersuchung) unterziehen müssen. In den offenen Teil der Krankenstation werden alle anderen Patientinnen, für die diese Voraussetzungen nicht zutreffen, eingewiesen. “ Mit $\$ 9$ wiederum wurde die Dauer des Aufenthalts an medizinische Notwendigkeiten gebunden: „Der Zeitraum des Krankenaufenthaltes einer Patientin richtet sich allein nach rein medizinischen Belangen, also nach der Dauer der erforderlichen Behandlung bzw. nach der Dauer der notwendigen Nachbeobachtung. “ 432

Im Folgenden werden einige Fragen zur Einweisung und zum Alltag auf der Krankenstation angesprochen. So sollten bei der Einweisung unter anderem die persönlichen Gegenstände gegen Quittung in Verwahrung genommen werden $(\$ 14)$. Mit den $\mathbb{S} 16$ bis 18 sollten alle Fragen des Arbeitseinsatzes geregelt werden. So heißt es im $\$ 16$ : „Die Patientinnen sind berechtigt, während der Dauer des Krankenhausaufenthaltes zu arbeiten." In der Freizeit sollten sich die Zwangseingewiesenen in der Bibliothek Bücher ausleihen können: „Für Freizeitbeschäftigung stehen ein Radio, Gesellschaftsspiele, sowie eine Bibliothek zur Verfügung. " 433 Grundsätzlich gab es keine Besuchszeit, wie es in $\$ 8$ heißt. Den Anweisungen des Arztes, des medizinisch-pflegerischen Per-

431 StAL: StVuR Nr. 7121. Hausordnung für die Krankenstation Thonberg (Anm. 430), Bl. 257.

432 StAL: StVuR Nr. 7121. Hausordnung für die Krankenstation Thonberg (Anm. 430), Bl. 258.

433 StAL: StVuR Nr. 7121. Hausordnung für die Krankenstation Thonberg (Anm. 430), Bl. 259. 
sonals, der Fürsorgerinnen und der Mitarbeiter der Verwaltung sollte „unverzüglich Folge“ geleistet werden (\$ 21). ${ }^{434}$

Des Weiteren waren in der Hausordnung eine Reihe konkreter Pflichten und Verbote geregelt: Jede Zwangseingewiesene sollte einmal wöchentlich duschen (\$27), Fußbäder und Kopfwäschen sollte genehmigt werden (\$28), und das Rauchen war laut Hausordnung nur in den Aufenthaltsräumen erlaubt (\$29). Darüber hinaus sollten sich die Zwangseingewiesenen tagsüber grundsätzlich in den Tagesräumen aufhalten ( $\$ 30)$ bzw. war der Aufenthalt auf den Fluren, Gängen und Treppen prinzipiell nicht erlaubt (\$31). Auch der Aufenthalt in den Schlafsälen war am Tag nicht gestattet, sofern nicht Bettruhe verordnet war (\$32). Schließlich war es nach der Hausordnung untersagt, sich in Krankenbekleidung auf oder in die Betten zu setzen bzw. zu legen ( $\$ 33$ ) oder bei geöffneten Fenstern Gespräche mit anderen Bewohnerinnen zu führen (\$ 34). 435

Für den Fall, dass diese Anweisungen nicht eingehalten wurden, sollten die Zwangseingewiesenen dies untereinander ansprechen, wie es im $\mathbb{} 22$ formuliert ist: „Die Patientinnen sollen sich zueinander rücksichtsvoll verhalten. Sie sind aber auch berechtigt, andere Patientinnen, die gegen die Disziplin verstossen, im Kollektiv zur Rede zu stellen und zur Einhaltung der Disziplin zu verpflichten. “ ̈̈hnlich wie in Halle (Saale) war auch die Instanz der Stubenältesten in der Hausordnung vorgesehen. Diese sollte die Zwangseingewiesenen überwachen, wie es im $\$ 23$ heißt: „Insbesondere haben auch die eingesetzten Vertrauenspatientinnen (Stubenalten) für Ordnung zu sorgen, auch deren Anweisungen sind von den Patientinnen zu befolgen." Diese Stubenältesten sollten als Scharnier zwischen dem medizinisch-pflegerischen Personal einerseits und den Zwangseingewiesenen andererseits fungieren: „Die Vertrauenspatientinnen sind als Sprecher der Patientinnen berechtigt Wünsche und Beschwerden vorzutragen " (\$ 24). 436

Schließlich wurden in der Hausordnung der Krankenstation Thonberg empfindliche Strafen aufgeführt. So sollten nach $\$ 25$ die Zwangseingewiesenen für „mutwillig verursachte Schäden“ haftbar gemacht werden. Laut $\$ 37$ konnte bei „schwerwiegenden Verstössen gegen die Hausordnung“ der leitende „Arzt die Verlegung der jeweiligen Patientin in ein verschliessbares Einzelzimmer“ anordnen. Schließlich heißt es im $\$ 38$ : „Wer während des Krankenhausaufenthaltes Straftaten, wie Körperverletzung und Sachbeschädigung begeht, wird zur Anzeige gebracht und gerichtlich zur Verantwortung gezogen. “437

Dass sich trotz der „Hausordnung für die Krankenstation Thonberg“ wenig an der Einweisungspraxis und dem Alltag auf der geschlossenen Station änderte, zeigen unter anderem die Aussagen einer Zeitzeugin, die 1971 „auf dem Bahn-

434 StAL: StVuR Nr. 7121. Hausordnung für die Krankenstation Thonberg (Anm. 430), Bl. $258 f$.

435 StAL: StVuR Nr. 7121. Hausordnung für die Krankenstation Thonberg (Anm. 430), Bl. $260 f$.

436 StAL: StVuR Nr. 7121. Hausordnung für die Krankenstation Thonberg (Anm. 430), Bl. $259 f$.

437 StAL: StVuR Nr. 7121. Hausordnung für die Krankenstation Thonberg (Anm. 430), BI. 261. 
hof aufgegriffen “ wurde. Dort hielt sie sich auf, weil sie viel Zeit im Zeitkino verbrachte, das am Bahnhof von Leipzig war. „Und dann war der Tag, als ich 13 Jahre alt gewesen bin und die Polizei mich wieder aufgefunden hat und mich dann anschließend erst aufs Revier und dann anschließend in den ,Barkas" nannte man das, reingetan hat, auch ein bisschen hart behandelt hat." "438 Eine Teilung der Station, wie in der Hausordnung vorgesehen, war Anfang der 1970er Jahre nicht erfolgt. Entsprechend fand die Aufnahme in die geschlossene Venerologische Station statt. Die Zeitzeugin beschreibt die Aufnahme aus ihrer Perspektive: „Und dann haben wir dann Einheitskleidung bekommen und dann mussten wir ja schon zur ersten Untersuchung, wo die Polizei dann, sage ich jetzt mal, so glaube ich, schon weg gewesen ist und da wurden dann die Haare geschnitten. Da hat man geweint. "Während der ersten Untersuchung erhielt die Zeitzeugin sofort eine Provokationsspritze: „Und dann haben die wogegen gespritzt.“ Die gynäkologische Erstuntersuchung aber auch die täglich folgenden Untersuchungen wurden sehr unsanft durchgeführt: Das ist „ganz grob gemacht worden (...). Wir wurden ignoriert. Wir sind strafend angeguckt worden, also richtig strafend angeguckt worden." 439 Entkleiden, Waschen, Abgabe der Wertgegenstände, Anlegen der Anstaltskleidung, gynäkologische Untersuchung und Abschneiden der Haare gehörten auch in Leipzig-Thonberg zur Prozedur der Aufnahme. Die täglichen Routinen auf der geschlossenen Venerologischen Station in Leipzig-Thonberg verliefen ähnlich wie in Halle (Saale): Aufstehen, medizinische Behandlung, Frühstück und Arbeitseinsatz. Täglich wurden die Zwangseingewiesenen um 6 Uhr morgens geweckt. „Dann mussten wir die Betten ordentlich machen, wie das so war, waschen gehen, alles unter Zeitdruck. Weil, wir hatten da bestimmte Zeiten dazu. "Nach „der Untersuchung, da sind wir dann forsch auch wieder zurück in die Zimmer. Da mussten die anderen Kinder oder Frauen Räume putzen. Und da haben wir auch unter anderem solches Besteck sauber gemacht. Ja, da haben wir so Schüsseln zum Abwaschen bekommen. “ ${ }^{400}$ An dieser Stelle wird ein weiteres Mal die Differenz zwischen der Hausordnung und der täglichen Routine deutlich. War in der Hausordnung die Arbeit freigestellt, berichtet die Zeitzeugin, dass sie arbeiten musste. Die Zwangseingewiesenen hatten eine interne Hierarchie entwickelt, in der vor allem die jungen Mädchen ganz unten waren. „Ohne Gefühle, man wurde dann eher von den älteren Mitstreitern dort geschubst und geschlagen. Ich habe mich da auch gewehrt." An solchen Übergriffen waren auch die sogenannten Stubenältesten beteiligt, die in der Hausordnung vorgesehen waren: „[J]a die Stubenälteste, die uns auch geschlagen hat, wenn wir das Brot für Wein nicht gegeben haben. (...) Der Wein, da mussten wir das Brot hingeben. Ich kann mich noch erinnern, auch wie die dasaß, die Hände so auf den Schoß und hat uns

438 Interview mit Frau LPC, geführt von Maximilian Schochow, Halle (Saale), den 20. Juli 2015.

439 Interview mit Frau LPC (Anm. 438).

440 Interview mit Frau LPC (Anm. 438). 
ganz böse angeguckt, ,Los, gibt deins her. Wir wollen da was machen, die setzen wir an.' Da haben die Wasser draufgemacht. Das haben die dann ins Fenster in die Sonne gestellt und da fing das dann schon nach einem Tag so ganz leicht an schaumig zu werden und das hat dann auch nach zwei Tagen ganz sauer gerochen. Das war wohl dann Wein. Das habe ich richtig noch im Kopf und das haben die getrunken." ${ }^{441}$

Selbst bei den täglichen Routinen zeigen sich also Unterschiede zwischen der festgelegten Hausordnung und der Praxis. Dies legt den vorläufigen Schluss nah, dass die Hausordnung vor allem den Versuch darstellte, die Einweisung und den Alltag formal rechtskonform und juristisch abgesichert zu gestalten. So war die Hausordnung vor allem eine verzögerte Reaktion auf die staatsanwaltlichen Ermittlungen im Jahr 1964. Dies zeigen die ersten Paragraphen deutlich. Dort wurden ausschließlich die rechtlichen Komponenten dieser Einrichtung und ihrer Funktion detailliert geregelt. Die Detailbesessenheit, mit welcher Sieler den rechtlichen Rahmen ausarbeitete, sollte formal mehr Rechtssicherheit bei künftigen Zwangseinweisungen bieten. Dies zeigt auch die Tatsache, dass noch im Januar 1967 eine klare Trennung der beiden Stationen in eine offene und eine geschlossene Station nicht umgesetzt war: „Diese Auflage konnte bis jetzt nicht realisiert werden, da die erforderlichen baulichen Vorhaben bisher nicht durchgeführt wurden. “442 Im März desselben Jahres sah eine weitere Planung die „Umprofilierung der Betten in der Krankenstation Thonberg" wie folgt vor: In der offenen Station sollten 38 Betten eingerichtet und in der geschlossenen Station 27 Betten vorgehalten werden. ${ }^{443}$ Entsprechend wurden die widerrechtlichen Zwangseinweisungen mindestens bis ins Frühjahr 1967 fortgeführt. Und selbst für die 197oer Jahren wurde diese Praxis von den Zeitzeuginnen beschrieben.

441 Interview mit Frau LPC (Anm. 438).

442 StAL: StVuR Nr. 7121. Medizinalrat H. Sieler. An Herrn Kreisarzt Med. Rat Dr. Gemkow. Besprechung. Leipzig, den 5. Januar 1967, Bl. 248.

443 StAL: StVuR Nr. 7121. Rat der Stadt Leipzig. Bericht über die Besprechung zu Fragen „Thonberg“ und „Lützowstr.“. Leipzig, den 21. März 1967, Bl. 247. 


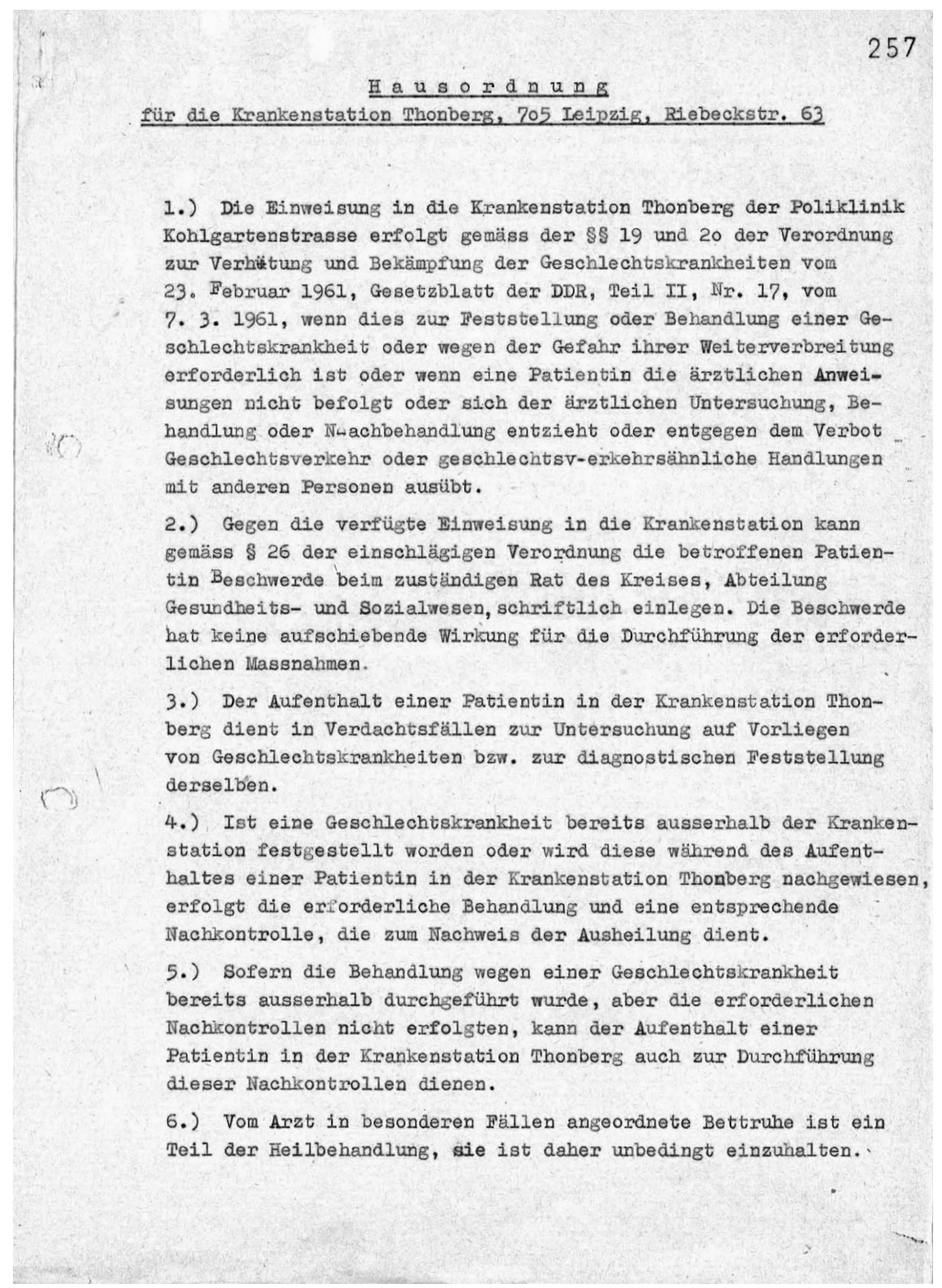

Abb. 14 Hausordnung für die Krankenstation Thonberg (1966) 


\subsection{Die Hausordnung der Krankenstation Thonberg}

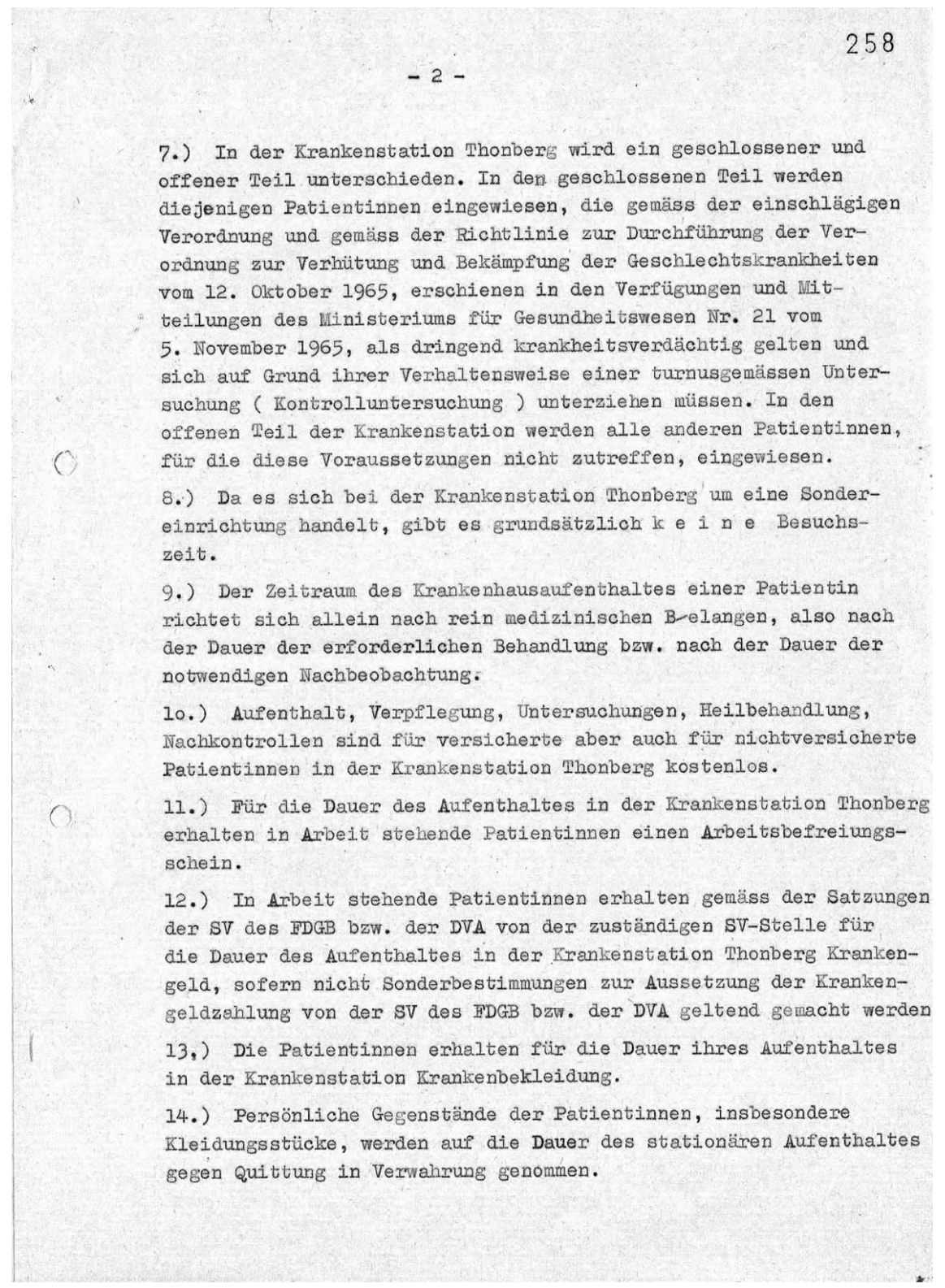

Fortsetzung von Abb. 14 Hausordnung für die Krankenstation Thonberg (1966) 


\section{Die geschlossene Venerologische Station in Leipzig-Thonberg}

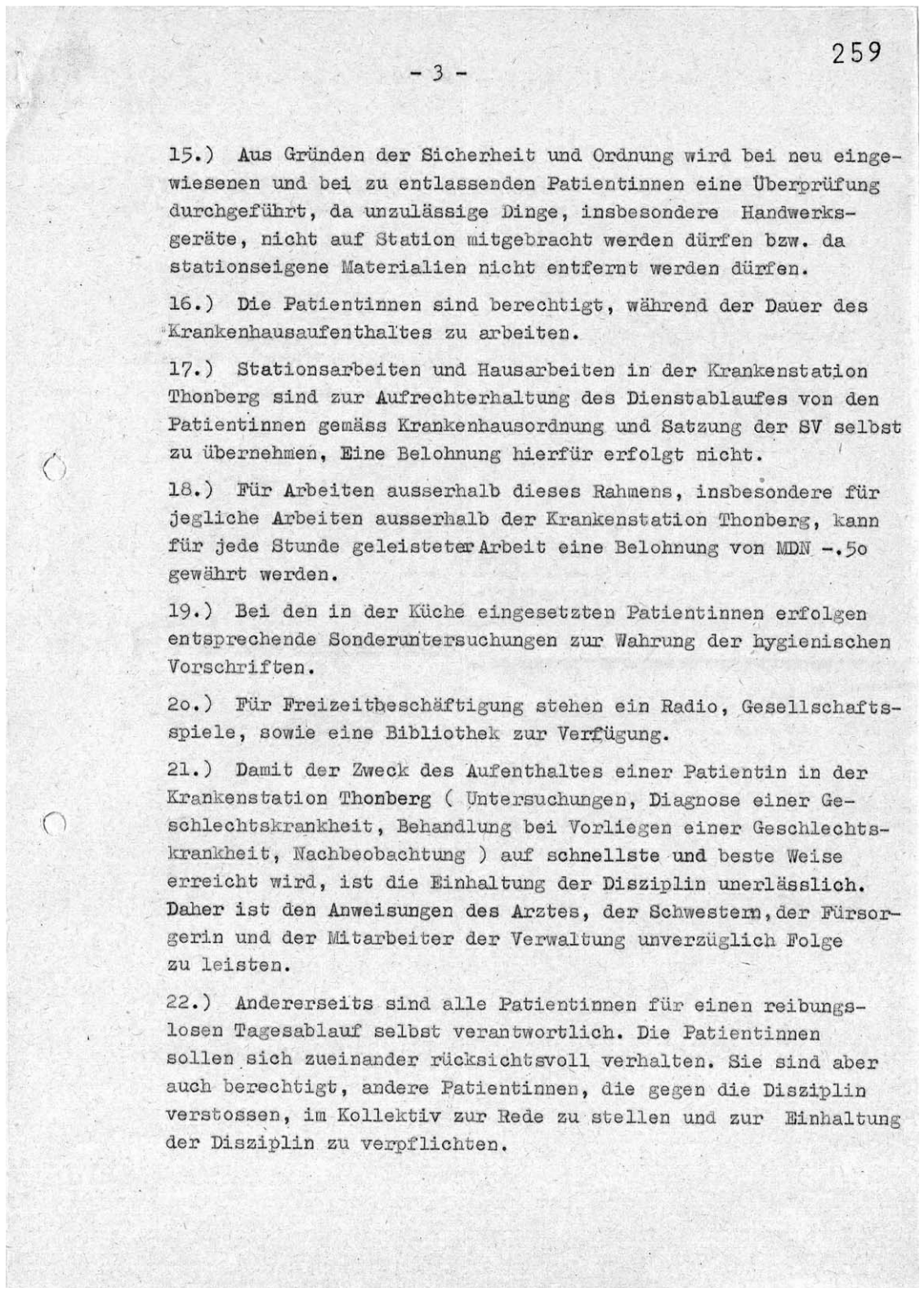

Fortsetzung von Abb. 14 Hausordnung für die Krankenstation Thonberg (1966) 


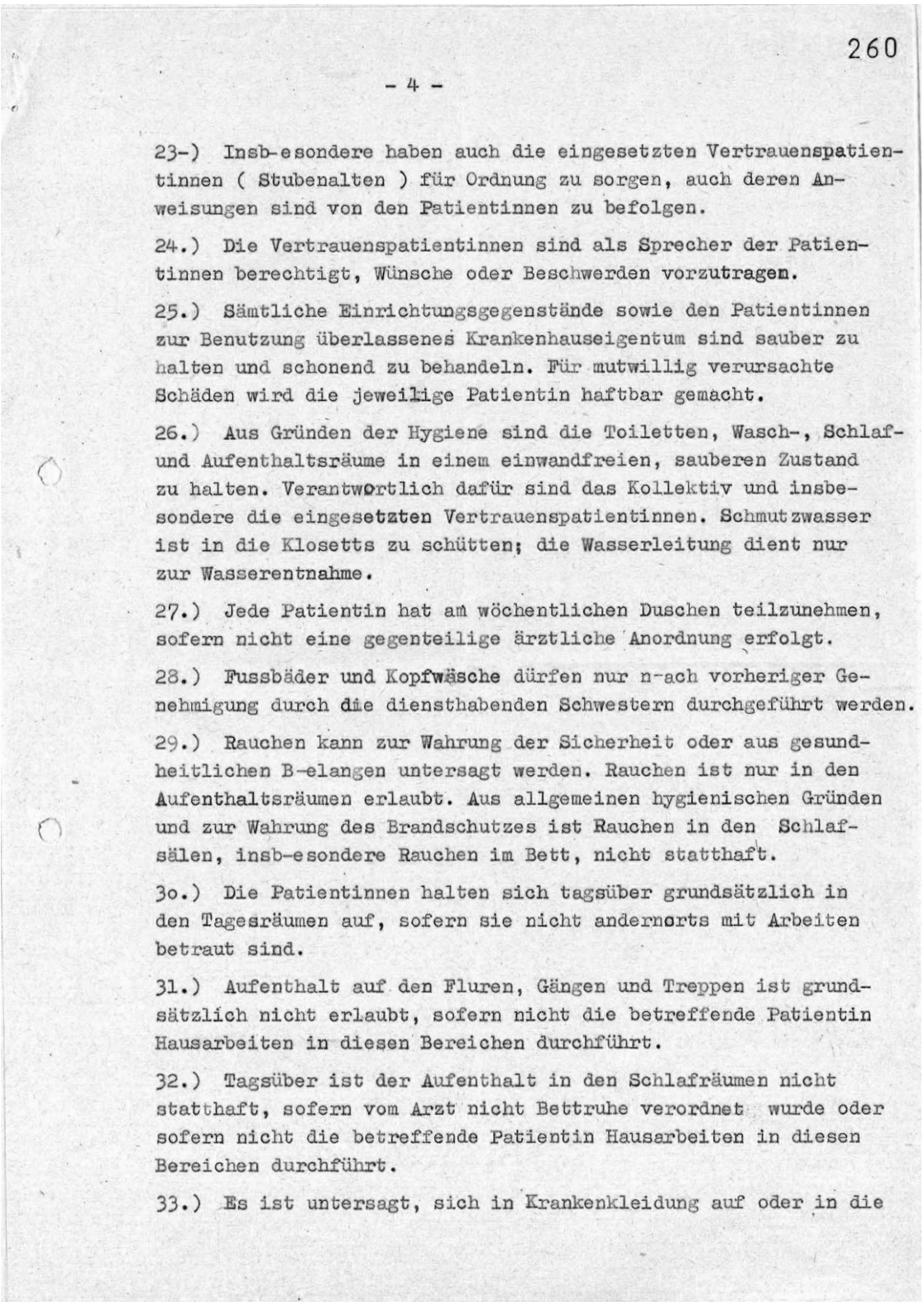

Fortsetzung von Abb. 14 Hausordnung für die Krankenstation Thonberg (1966) 


\section{Die geschlossene Venerologische Station in Leipzig-Thonberg}

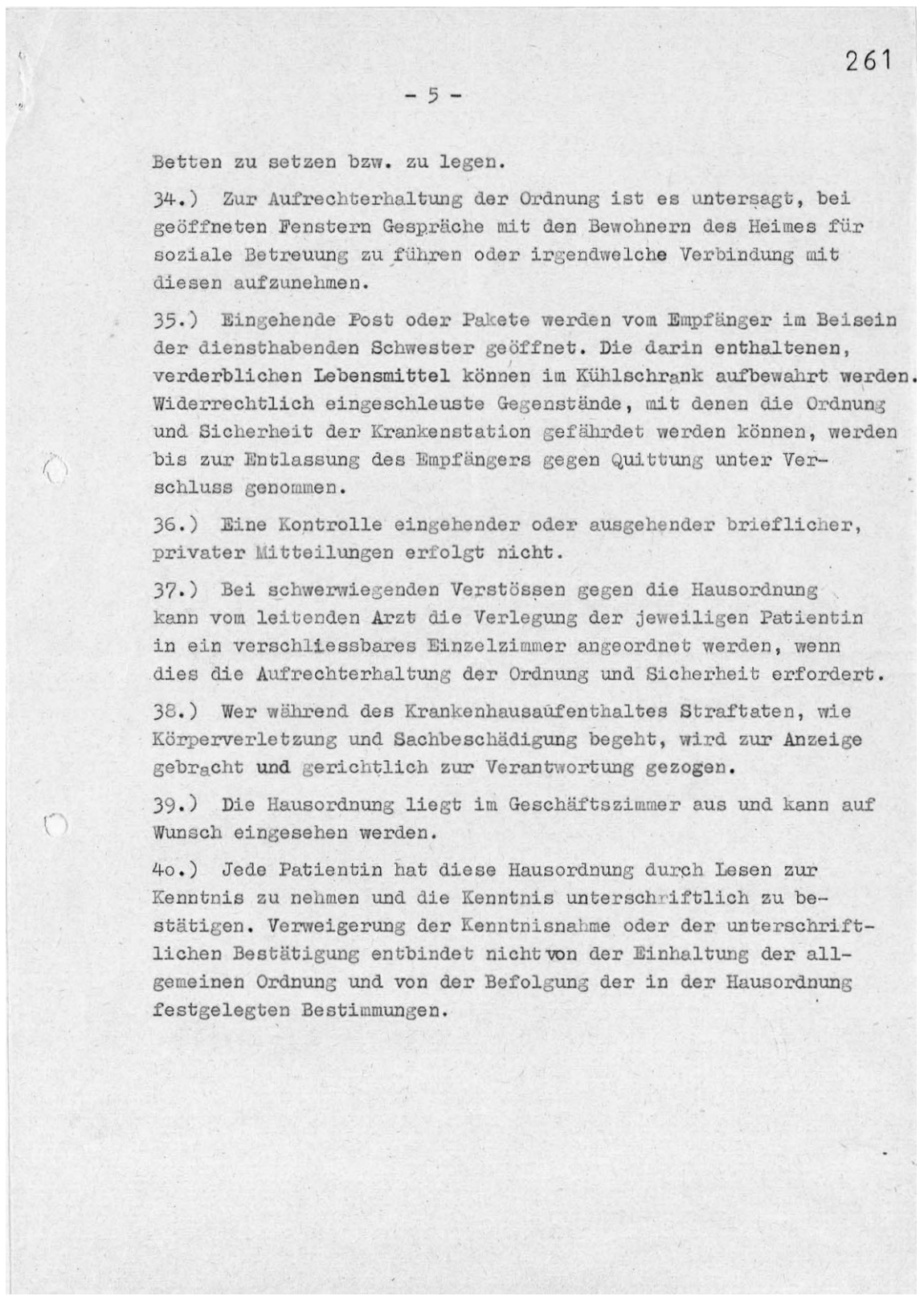

Fortsetzung von Abb. 14 Hausordnung für die Krankenstation Thonberg (1966) 


\subsection{Die geschlossene Krankenstation Thonberg in den 1970er Jahren}

Die Abweichungen der Praxis von der Hausordnung machten sich jedoch nicht nur bei der Einweisung in die Station bemerkbar. Eine Zeitzeugin, die 1971 in der geschlossenen Venerologischen Station von Leipzig-Thonberg war, berichtet: „Ich war in Leipzig auf dem Hauptbahnhof und es war halt so, wenn da junge Mädchen sich irgendwie, na sag ich mal, wie die gesagt haben, sich rumgetrieben haben oder so, da wurden die aufgegabelt von der Transportpolizei und dann wurdest du da hingeschafft, ja. Es hieß dann erst einmal: ,Sie kommen erst einmal auf eine Station. 'Keine Ahnung, warum, ja. Ja, und da haben die mich nach Leipzig, nach dem Thonberg gefahren, ja, und da war ich dort auf der Station. Und da kam ich halt dort an und dann wurde als Erstes eine Untersuchung gemacht. Also das heißt, eine frauenärztliche Untersuchung, ja. Mit Abstrich und alles was so dazugehört. Und dann kamst du halt praktisch in so eine Art wie Gruppenraum, Zelle oder wie immer man das sagt, größerer Raum, wo mehrere Patientinnen waren. Alles junge Mädchen, zum Teil 14-, 15-Jährige, wo man sich fragt, mein Gott, was machen die mit den Kindern, sozusagen, ja. Ich muss ehrlich sagen, zu der Zeit hab ich mir noch nie so viele Gedanken gemacht. Diese Gedanken darüber, was uns dort alles passiert ist, die sind erst viel später gekommen. Es hieß dann halt, wir müssen hier drinnen bleiben, es ist eine Klinik, ja. Ja, und dann hast du praktisch, wenn man da überhaupt keine Ahnung hat, was eigentlich mit einem passiert. Man weiß überhaupt nicht, was man sagen soll. Dann hast du halt auch Leute dabei gehabt, die schon länger da waren, die praktisch dich dann sozusagen eingewiesen haben, was es hier mit der Station auf sich hat. (...) Früh musste man als Erstes, bevor man Frühstück bekam, musste man erst zur Untersuchung. Also, wir standen da in Reih und Clied vor der Tür von dem Doktor und dann mussten wir halt da zur Untersuchung. Und ich kann mich noch entsinnen, es war so ein ganz junges Mädchen, die war gerade mal so 14, die hat so bitterlich geweint da drinnen, weil sie sich erstens wahrscheinlich sicherlich geschämt hat und zweitens, weil sie Angst hatte auch, ja und die hat richtig geschrien, ja. Ich habe das dann erst viel später erfahren, was eigentlich da drinnen so los ist, ja. Also ich sag mal, das Schlimmste war halt, dass du jeden Tag, jeden Tag zu der Untersuchung musstest. Dann kriegtest du dein Frühstück, ja, und dann musstest du halt in der Cruppe dableiben, ja. Also, es war nicht so, dass man da irgendwie, ja, eine Beschäftigung hatte oder etwas Nützliches machen konnte, gar nicht. Man saß halt dort und verbrachte die Zeit praktisch ja, indem man sich mit allen anderen beschäftigte, ja. " 444

In dieser Beschreibung wird noch einmal deutlich, wie jung die Mädchen waren, die in Leipzig-Thonberg Anfang der 1970er Jahre zwangseingewiesen wurden. Nicht nur in Halle (Saale), sondern auch in Leipzig-Thonberg waren die

444 Interview mit Frau LPD, geführt von Florian Steger und Maximilian Schochow, Halle (Saale), den 11. November 2013. 
Mädchen teilweise nicht älter als 13 oder 14 Jahre. Zudem wirken die Aussage der Zeitzeugin aus Halle (Saale) und die Beschreibung der ehemaligen Patientin in Leipzig-Thonberg wie Spiegelbilder. Es wird deutlich, dass in Halle (Saale) und in Leipzig Mädchen und Frauen von der Polizei aufgegriffen und ohne Beachtung der „Verordnung zur Verhütung und Bekämpfung von Geschlechtskrankheiten “ ${ }^{445}$ sofort zwangseingewiesen wurden. Die Mädchen wurden von der Transportpolizei auf öffentlichen Plätzen, meist dem Hauptbahnhof, festgenommen und sofort eingewiesen. Eine Zeitzeugin ergänzt: „Es war ja zu DDR-Zeiten wirklich so, dass regelrecht Jagd auf junge Mädchen gemacht wurde. Das ist Fakt. Ja, also, wenn du als junges Mädchen alleine auf dem Bahnhof warst, warst du für die ein Rumtreiber, egal, und wenn du noch dazu keine Fahrkarte hattest, dann warst du geliefert, ja, ob das in Weißenfels war oder in Halle oder in Leipzig. Das war überall so, ja. “"46 Eine weitere Zwangseingewiesene, die Ende der 197oer Jahre in Leipzig-Thonberg war, wurde ebenfalls von der Polizei aufgegriffen. 1977 erlebte sie die Festsetzung wie folgt: „Meine Freundin war dabei, ja und wir wurden dann erst einmal aufs Revier gebracht und da wurden wir dann da reingeführt und wurden in eine Zelle eingesperrt, also jeder in eine Extrazelle und saßen da halt drinnen." Anschließend wurde sie vom Revier in die Kohlgartenstraße und dann in die geschlossene Venerologische Station überführt. ${ }^{477}$ Diese Schilderungen zusammengenommen offenbaren den anhaltenden Verstoß gegen die 1961 erlassene „Verordnung zur Verhütung und Bekämpfung von Geschlechtskrankheiten“448 bzw. die Ausführungen des Leipziger Staatsanwalts Fräbel. Die Zeitzeuginnen wurden nicht zwangseingewiesen, weil sie auf einer HwG-Liste standen. Vielmehr wurden sie während der gesamten 197oer Jahre auf der Straße aufgegriffen oder in öffentlichen Räumen festgesetzt und in die geschlossene Krankenstation Leipzig-Thonberg eingewiesenen.

Die Aufnahmeprozedur und die täglichen Routinen in Leipzig-Thonberg glichen denen in Halle (Saale) ebenso wie die täglichen schmerzhaften Untersuchungen: „Na da wurde einfach ein Glasrohr eingeführt. Also da kann ich mich halt auch daran erinnern, dass es schon ziemlich schmerzhaft war, weil ich habe auch immer runtergeguckt, was liegen für Röhrchen da, was nimmt sie für eins und das war unterschiedlich. " Die gynäkologischen Untersuchungen und die Abstriche wurden meist von den Pflegerinnen durchgeführt: „Es gab verschiedene Schwestern ja. Also es gab eine, die war sehr, sehr böse." So blieb der Zeitzeugin, die Ende der 197oer Jahre in Leipzig-Thonberg war, folgende Begebenheit in Erinnerung: „Was mir noch einfällt, noch eine Erinnerung, die ich auch nie vergessen habe, auf dem Thonberg, da war ein Mäd-

445 Verordnung zur Verhütung und Bekämpfung von Geschlechtskrankheiten (Anm. 23).

446 Interview mit Frau LPD (Anm. 444).

447 Interview mit Frau LPE, geführt von Florian Steger und Maximilian Schochow, Halle (Saale),

den 5. November 2014.

448 Verordnung zur Verhütung und Bekämpfung von Geschlechtskrankheiten (Anm. 23). 
chen, die wurde bei uns eingeliefert. (...) Die nannten wir Atze, weil die sich immer, die hat immer so rote Bäckchen gehabt und die war auch so unerfahren und als Spitznamen haben wir die so genannt. Wie die richtig heißt, weiß ich nicht mehr. Und die hatte noch nie einen Mann (...), die wurde trotzdem untersucht und der ging es richtig dreckig. Die hat geschrien, die hatte Angst. Die haben dann die Tür zugemacht, weil die Tür war ja immer offen, wenn wir gestanden haben. Da ging die erste rein, die kam raus, die nächste geht rein und dann war sie dran. Die wusste ja noch nicht mal, wie sie sich auf so einen Stuhl legt, so ungefähr. Und da habe ich es bloß klatschen gehört. Das waren dann die Stubenalten wahrscheinlich, die haben da, also haben wir dort gesehen, wie sie der die Beine auseinandergerissen haben und immer draufgeklatscht haben und die wurde dann untersucht. Und die saß dann bloß noch da irgendwie und war total fertig und die war dann irgendwann weg, die war nicht mehr da." 449

Anfang der 1970er Jahre, so die Aussagen ehemaliger Patientinnen, wurden die Zwangseingewiesenen noch beschäftigt. Ende der 197oer Jahre gab es kaum noch Beschäftigung: „Also das war einfach, da ist nicht viel passiert. Also das war einfach nur warten. (...) Wir hatten nichts da, womit wir uns beschäftigen konnten, also wir hatten keine Bücher, wir hatten kein Fernseher, kein Radio. “450 Diese Aussage zeigt auch, dass die in der „Hausordnung für die Krankenstation Thonberg “ vorgesehene Bibliothek in den 1970er Jahren nicht existierte. Es wurde einfach auf das Essen gewartet. Diese Langeweile führte zu Aggressionen und Gewalt unter den Zwangseingewiesenen: „[I]ch habe viele Schlägereien miterlebt. Ich habe miterlebt, wie Frauen zusammengeschlagen wurden, ich habe auch einmal mitgeschlagen, weil ich mitschlagen musste. Ich selber bin nicht geschlagen worden. "Ende der $1970 e r$ Jahre wurde nur einer der beiden Säle in der zweiten Etage genutzt: Die Frauen waren alle „in einem Raum. Die waren alle in einem Raum. (...) Es waren, ich glaube, also über 30 waren das schon, weil ich habe mir das immer so gemerkt: Ich war 30 Tage da. " 451 Für diese Zeit sind auch Ausbrüche aus der geschlossenen Venerologischen Station in Leipzig-Thonberg überliefert: „Und dann, was mir auch noch, an was ich mich auch noch erinnern kann: zwei Mädels sind ausgerissen, die haben sie dann aber wieder geschnappt, also gefunden und wieder zurückgebracht, die mussten dann in die Einzelzelle. "452 Diese Strafmaßnahme war in der „Hausordnung für die Krankenstation Thonberg“ explizit vorgesehen und wurde, wie die Aussage der Zeitzeugin belegt, auch praktiziert. 453

Schließlich waren die Zwangseingewiesenen in Leipzig-Thonberg analog zur geschlossenen Venerologischen Station in Halle (Saale) dazu aufgefordert, bei

449 Interview mit Frau LPE (Anm. 447).

450 Interview mit Frau LPE (Anm. 447).

451 Interview mit Frau LPE (Anm. 447).

452 Interview mit Frau LPE (Anm. 447).

453 StAL: StVuR Nr. 7121. Hausordnung für die Krankenstation Thonberg (Anm. 430), BI. 261. 
der Entlassung eine Schweigeerklärung zu unterschreiben: „Ja, es hieß dann so ungefähr, wir können jetzt wieder gehen, ja, und dann musstest du da unterschreiben und du musstest unterschreiben, dass du das, was hier drinnen passiert ist, nicht weitererzählst, dass du Schweigepflicht hast, das mussten wir ja überall, ja." ${ }^{454}$ Eine solche Erklärung musste auch die Zeitzeugin unterzeichnen, die Anfang der 1970er Jahre in Leipzig-Thonberg war: „Am 22.1. bin ich entlassen worden, 1971, vier Wochen. (...) Ja ich habe hier etwas unterschrieben. Das ist auch dabei. Da musste ich als Dreizehnjährige unterschreiben. “455 Das Prinzip der Schweigeerklärung wurde bis Ende der 1970er Jahre angewandt. ${ }^{456}$ Zumindest an diesem Punkt wird eine Änderung im Vergleich zur Entlassung in den 196oer Jahren deutlich, als die Frauen und Mädchen bei ihrer Entlassung noch keine Schweigeerklärung unterzeichnen mussten. Dies zeigt, dass sich die rechtswidrige Praxis auf der geschlossenen Venerologischen Station in Leipzig-Thonberg durchsetzte und keine Ausnahme darstellte. Stattdessen wurde das System durch diese Erklärungen untermauert und erschwerte den Zwangseingewiesenen die Möglichkeit zum Widerspruch gegen das, was ihnen widerfahren war. Und noch ein Unterschied wird im Vergleich zu den 196oer Jahren deutlich: Die Zeitzeuginnen, die in den 1970er Jahren in Leipzig-Thonberg zwangseingewiesen waren, wurden vier Wochen in der geschlossenen Venerologischen Station festgehalten: „Ja, in Leipzig, war ich glaube ich, vier Wochen und dann durfte ich wieder nach Hause. “457 Auch eine weitere Zeitzeugin wurde Ende der 1970er Jahre vier Wochen zwangsbehandelt. ${ }^{458}$

Für die geschlossene Venerologische Station in Halle (Saale) konnten wir anhand der Interviews mit den Zeitzeuginnen und der Analyse der Hausordnung vier Funktionen für diese Station herausarbeiten: 1. Die Erziehung zu einer „sozialistischen Persönlichkeit“, 2. die arbeitstherapeutische Funktion im Sinn einer Pflicht zur täglichen Arbeit, 3. die Isolierung sowie 4. die medizinische Versorgung, soweit eine Krankheit vorlag. Diese Funktionen lassen sich für die Krankenstation Leipzig-Thonberg nur teilweise bestätigen.

Ein klarer Unterschied zwischen Halle (Saale) und Leipzig-Thonberg bestand in der Funktion der Erziehung. Die Erziehung der Zwangseingewiesenen, die in Halle (Saale) an erster Stelle stand, wurde in der „Hausordnung für die Krankenstation Thonberg " kaum verankert. Weder die gesetzestreue noch die politisch gefestigte Patientin waren ein Ziel der Hausordnung von LeipzigThonberg. Dennoch war Erziehung im Sinn einer Disziplinierung in der Hausordnung implementiert und Teil der täglichen Routine, wie aus den Aussagen der Zeitzeuginnen hervorgeht. Der Tagesablauf war detailliert festgelegt. Für

454 Interview mit Frau LPD (Anm. 444).

455 Interview mit Frau LPC (Anm. 438).

456 Interview mit Frau LPE (Anm. 447).

457 Interview mit Frau LPD (Anm. 444).

458 Interview mit Frau LPE (Anm. 447). 
die Disziplinierung sprechen auch die Pflichten, Verbote und Strafen, die in der Hausordnung geregelt waren und im Alltag angewandt wurden, wie die Zeitzeuginnen berichten. Über die Instanz der Stubenältesten wurden die konkreten Pflichten und Verbote im Alltag geregelt und überwacht - beispielsweise der Aufenthalt auf dem Flur. Darüber hinaus zeigt sich, dass die in der „Hausordnung für die Krankenstation Thonberg“ festgelegte Isolation in einem verschlossenen Einzelzimmer auch in der Praxis eingesetzt wurde. Hier werden noch einmal Überschneidungen mit Halle (Saale) deutlich. Auffällig ist im Vergleich, dass die Disziplinierung in Leipzig-Thonberg weniger konsequent vorangetrieben wurde. Während in Halle (Saale) ein ausgeklügeltes System von Belobigungen und drakonischen Strafen installiert wurde, etwa das Sitzen auf einem Hocker über 24 Stunden, sind derartige Strafen für Leipzig-Thonberg nicht überliefert. Solche Formen der Bestrafung kamen weder in der „Hausordnung für die Krankenstation Thonberg“ noch in den Beschreibungen der Zeitzeuginnen vor.

Auch hinsichtlich der arbeitstherapeutischen Funktion werden Unterschiede zwischen Halle (Saale) und Leipzig-Thonberg deutlich. In den 196oer Jahren mussten die Zwangseingewiesenen in Leipzig-Thonberg arbeiten, wie die Zeitzeuginnen berichten. Diese Pflicht zur Arbeit wird auch noch für die frühen 1970er Jahre beschrieben. In der „Hausordnung für die Krankenstation Thonberg " aus dem Jahr 1966 heißt es in $\$ 16$, dass die Zwangseingewiesenen berechtigt waren, während der Dauer des Krankenhausaufenthaltes zu arbeiten. Eine Pflicht zur Arbeit lässt sich daraus nicht ableiten. Dies bestätigen auch die Zeitzeuginnen, die Mitte und Ende der 197oer Jahre in Leipzig-Thonberg zwangseingewiesen waren. Sie mussten nicht mehr arbeiten. Vielmehr wäre die Arbeit für die ehemaligen Zwangseingewiesenen eine willkommene Abwechslung im monotonen Alltag gewesen. Entsprechend war die arbeitstherapeutische Funktion im Sinn einer Pflicht zur täglichen Arbeit nur in den 196oer und Anfang der 1970er Jahre gegeben. Diese Pflicht stand noch in der Tradition des Fürsorgeheims Leipzig-Thonberg, in dem täglich 8 Stunden gearbeitet werden musste, und wird mit der „Hausordnung für die Krankenstation Thonberg " formal ausgesetzt. In der täglichen Praxis der geschlossenen Venerologischen Station Leipzig-Thonberg wird in den 197oer Jahren aus der Pflicht zur Arbeit die Möglichkeit zur Beschäftigung. „Arbeitstherapeutische“ Zwecke sind in den $1970 e r$ Jahren mit den Beschäftigungen nicht verbunden.

Die Funktion der Isolation war sowohl für die geschlossene Venerologische Station in Halle (Saale) als auch für die geschlossene Krankenstation Thonberg gegeben. In beiden Stationen waren die Zwangseingewiesenen von der Außenwelt getrennt. In der „Hausordnung für die Krankenstation Thonberg“ waren Besuchszeiten explizit untersagt. Dass die Zwangseingewiesenen keinen Besuch empfangen durften, wurde auch in den Interviews deutlich. Zudem beschrieben die Zeitzeuginnen die Station als abgeschlossenen Ort, den sie nicht verlassen konnten. Diese Isolation führte immer wieder zu Ausbruchsversuchen und zu Ausbrüchen. 
Schließlich folgt die Funktion der medizinischen Versorgung. Die geschlechtskranken oder krankheitsverdächtigen HwG-Personen sollten untersucht werden, eine Diagnose erhalten und behandelt werden, wenn eine Geschlechtskrankheit festgestellt wurde. ${ }^{459}$ Dass diese in der Hausordnung beschriebene Funktion umgesetzt wurde, zeigen die Aussagen der Zeitzeuginnen für die 197oer Jahre eindrücklich. Die medizinische Versorgung war Teil der täglichen Routine. Ähnlich wie in Halle (Saale) wurde täglich in die Integrität der zwangseingewiesenen Mädchen und Frauen ohne Aufklärung und ohne Einwilligung eingegriffen. Täglich wurden Abstriche genommen und den Untersuchten dabei Schmerzen zugefügt.

Einen direkten Vergleich zwischen Leipzig-Thonberg und Halle (Saale) ermöglicht auch die Aussage einer Zeitzeugin, die 1971 in Leipzig-Thonberg war und zwei Jahre später in die geschlossene Venerologische Station in Halle (Saale) zwangseingewiesen wurde: „In Halle war es natürlich ein bisschen schlimmer wie in Leipzig. Wir sind dort, also man muss sich vorstellen, man wurde auch von Halle, von der Transportpolizei, genau wie in Leipzig, die kriegten den Auftrag, zack, in die Poliklinik. Und man denkt ja jetzt, du kommst dort in ein Krankenhaus, ja. Ja sicher, auch eine geschlossene Station. Ich kann Ihnen nicht mal mehr sagen, was da für Fenster waren. Ich weiß nur, dass Gitter davor waren. Also, es war auch eine geschlossene Station, vorne war alles zu und es war in der ersten Etage und also, hier war die Station, glaube, wenn man reinkommt links, war unsere Station und gerade rüber, über den Flur, war noch eine Station. (...) Das wussten wir aber nicht, ja, wir waren ja nur da drinnen eingeschlossen. Und dort war ich auch mit vier, fünf Leuten im Zimmer, ja. (...) Ja, und in Halle, das fällt mir heute noch schwer, darüber zu reden. Das war so schlimm. " Darüber hinaus schildert die Zeitzeugin, dass die Untersuchungen in Halle (Saale) "schlimmer“ als in Leipzig waren. „Ich hatte ihnen das ja erklärt mit den Röhrchen, ja. Dort war es halt auch so, da war auch so ein junges Mädchen also, (...), Wenn die heult, drehe ich noch weiter rein.' (...) Der hat praktisch, wenn Sie so ein Röhrchen reinkriegen und das war ja nicht gerade bloß so kurz, ja, dann geht das praktisch bis zur Gebärmutter und dann hat der dann so richtig gedreht. Ja, und die haben natürlich geschrien, die kleinen Mädchen, ja. "Auch nach der Entlassung aus der geschlossenen Venerologischen Station in Halle (Saale) musste die Patientin eine Schweigeerklärung unterzeichnen, sich bei der Fürsorgestelle in ihrem Heimatort melden und, wie alle ehemaligen Zwangseingewiesenen, zu den wöchentlichen Nachuntersuchungen erscheinen. ${ }^{460}$

459 StAL: StVuR Nr. 7121. Hausordnung für die Krankenstation Thonberg (Anm. 430), Bl. 259. 460 Interview mit Frau LPD (Anm. 444). 


\subsection{Die Asylierung Geschlechtskranker in der geschlossenen Venerologischen Krankenstation Thonberg in den 1980er Jahren}

In den 1980er Jahren war die geschlossene Venerologische Krankenstation Thonberg eine der letzten Stationen ihrer Art im Süden der DDR. Bereits 1982 war die geschlossene Venerologische Station der Poliklinik Halle (Saale) aufgelöst worden und der dortige Terror hatte ein Ende (Kap. 4.8). Für die Station in Leipzig-Thonberg hatte die Auflösung der Station in Halle (Saale) zudem die Konsequenz, dass unliebsame und undisziplinierte Zwangseingewiesene nicht mehr nach Halle (Saale) überwiesen werden konnten. Überhaupt zeichnete sich in den 1980er Jahren ein Wandel in der geschlossenen Venerologischen Krankenstation Thonberg ab, in dessen Folge die zwangseingewiesenen Mädchen und Frauen vor allem asyliert wurden.

Trotz der Veränderungen lassen sich eine Reihe von Kontinuitäten beschreiben: Auch in den 1980er Jahren wurden in Leipzig Mädchen und Frauen von der Polizei auf der Straße oder öffentlichen Plätzen festgesetzt und in die geschlossene Venerologische Krankenstation Thonberg gebracht. Eine Zeitzeugin, die zu dieser Zeit in Leipzig-Thonberg zwangseingewiesen wurde, erinnert sich: „, [I]ch hatte eigentlich nur kein Fahrgeld gehabt für ins Jugendwohnheim zu fahren, da sind wir natürlich getrampt, also bin ich getrampt, sollte ich dahin laufen? Ja, und dann hat man mich da aufgefangen. Die Polizei hat mich dann zur nächsten Wache gefahren, wo ich 24 Stunden bleiben musste und dann kam ich ins Durchgangsheim, war ich drei Tage in der Zelle, ja und dann sollte ich nach Thonberg so oder irgendwie so eine Einrichtung, wo ich noch nicht wusste, was es für eine Einrichtung war und dann konnten sie mich aber nicht aufnehmen, weil noch keine Voruntersuchung stattfand, so und bei der Voruntersuchung stellten sie fest, dass ich gesund war, so und trotzdem haben sie mich dahin geschleppt ja. (...) und ja, abends bin ich ja dann in den Thonberg gekommen. " 461

Eine weitere Zeitzeugin, die Mitte der 1980er Jahre in die geschlossene Venerologische Krankenstation Thonberg kam, erlebte die Einweisung ähnlich: „Also ich war mit Bekannten in Leipzig eigentlich auf dem Rummel.“ Gegen 22 Uhr wurde sie von der Polizei auf dem Markt von Leipzig angehalten. „Und da hat die Polizei mich aufgehalten und hat gesagt, ich muss mitkommen und weg war ich. (...) die Polizei, die hat mich halt angehalten und ich war mit anderen Leuten zusammen, und dann ging es gleich zu einer ärztlichen Untersuchung (...). Die haben einen irgendwo hingefahren zum Arzt und der hat gleich gesagt, also ich bin nicht daran erkrankt, das weiß ich noch und dann haben sie aber gesagt, um sicher zu gehen, muss ich noch eine Nachuntersuchung machen am anderen Tag und da haben sie mich auch noch dann dorthin gefahren (...) es hieß halt, um die Bestätigung zu kriegen, dass nichts

461 Interview mit Frau LPF, geführt von Maximilian Schochow, Halle (Saale), den 13. April 2015. 
ist, müsste ich morgen nochmal untersucht werden und da sind sie mit mir dahin gefahren und naja, da hat man dann gleich seine Sachen weggenommen gekriegt und wir sind in so einen Waschraum, Gemeinschaftswaschraum gekommen und dann war man erstmal da drin." ${ }^{462}$ Diese Schilderung wird durch die Krankenakte der Zeitzeugin bestätigt, die in der geschlossenen Venerologischen Krankenstation Thonberg geführt wurde. Das Stadtarchiv Leipzig übergab der ehemaligen Patientin diese Akte, aus der auch hervorgeht, dass die Zeitzeugin mit „Verdacht auf eine Trichomoniasis“ eingeliefert wurde. Dieser Verdacht bestätigte sich im Verlauf ihres Aufenthalts auf der geschlossenen Venerologischen Krankenstation Thonberg jedoch nicht. ${ }^{463}$

Eine ehemalige ungelernte Pflegekraft, die von 1988 bis 1990 auf der geschlossenen Station tätig war, erinnert sich an die Einweisung und Aufnahme dort: „Also das war so, dass dort eigentlich kein Patient freiwillig hinkam. Also mir ist auch nicht erinnerlich, dass da jemand freiwillig hingekommen ist. Die wurden eigentlich alle gebracht. In der Regel von der Polizei. " ${ }^{64} 4$ Somit bestätigt die Zeitzeugin, dass die Mädchen und Frauen unter Zwang auf die geschlossene Venerologische Station kamen und auch noch Ende der 198oer Jahre durch die Polizei zugeführt wurden. Bei der Aufnahme der Mädchen und Frauen war die Unterstützung der Hilfsschwestern gefragt: „Also das war so gewesen, dass die Patienten alle ihre Sachen abgeben mussten. Die wurden dann halt auf Ungeziefer, kann man so sagen, untersucht, also Flöhe und so. Also da mussten die sich komplett entkleiden, wurden dann von oben bis unten untersucht. Also so ähnlich wie ein Dermatologe das machen würde, außer, wir haben nicht so auf Hauterkrankungen geschaut, sondern eher auf solche Sachen und dann mussten die duschen. Das war nicht immer ganz angenehm, weil oftmals die Warmwasserversorgung ausgefallen ist. Die mussten dann das aber trotzdem machen." 465

Wie in den 1970er Jahren wurden die Zwangseingewiesenen auch in den 1980er Jahren unmittelbar nach der Einweisung in die geschlossene Venerologische Krankenstation Thonberg gynäkologisch untersucht, wie eine ehemalige Zwangseingewiesene berichtet: „Ja, dann bin ich dort angekommen und musste ich auch gleich ins Untersuchungszimmer zur Aufnahme, danach musste ich mich komplett ausziehen. Der Arzt kontrollierte erst mit seinen Händen und Fingern an meinen Körperöffnungen, was auch sehr wehtat. Aber es interessierte den ja gar nicht, danach musste ich auf den Untersuchungsstuhl, fixierte meine Beine unten fest mit Schlingen, damit ich die nicht wieder zusammenkriege, ja und dann hielt er sie auseinander und die Schwester, die drückte meinen Oberkörper am Stuhl ran, damit ich mich nicht

462 Interview mit Frau LPG, geführt von Florian Steger und Maximilian Schochow, Halle (Saale), den 16. November 2014.

463 Interview mit Frau LPG (Anm. 462).

464 Interview mit Frau LSA, geführt von Maximilian Schochow, Halle (Saale), den 22. April 2015.

465 Interview mit Frau LSA (Anm. 464). 
wehren konnte. Ja und dann nahm er so ein dickes Glasrohr und rammte es mir tief in den Unterleib rein. Klar schrie ich vor Schmerzen und da lief auch gleich das Blut raus, weil er zu doll damit gearbeitet hatte, ja, und als er fertig war, da suchte er meinen Körper, also meine Kopfhaut nach Läusen ab, so als Vorsichtsmaßnahme, und mir wurden auch nachher von der Schwester die Haare geschoren, ja und das ging fast jeden Tag so, ja, also vier Wochen lang Untersuchung. " Nach der Untersuchung musste die Zeitzeugin ihre persönlichen Sachen abgeben und Anstaltskleidung anziehen: „Ja, ich hatte ja nichts weiter gehabt, außer meine Klamotten am Leib." Anschließend wurden ihr die Haare abgeschnitten: „Die wurden auch abgeschnitten ja, zwar nicht allzu kurz, Igel oder so, aber die wurden schon ziemlich kurz abgeschnitten, ja. “ Wenn die Mädchen und Frauen nachts zugeführt wurden, fand die gynäkologische Erstuntersuchung erst am Morgen des folgenden Tages statt: „Ich bin dann erstmal eingesperrt worden im Waschraum und sind die ganzen Sachen weggenommen worden. (...) Auch die Anziehsachen alles, haben sogar gesagt die ganze Unterwäsche und dann hat es eine Ewigkeit gedauert und dann haben sie mich dann in den Schlafraum gebracht. “466

Der Tag begann auch in den 1980er Jahren um 6 Uhr morgens. „Also wir sind ungefähr um 6 aufgestanden. Ja und dann mussten wir zur Untersuchung gegen $7 \mathrm{Uhr},(. .$.$) wir mussten uns gleich dort vorm Untersuchungszimmer im$ Schlafhemd, ohne Unterhosen mussten wir uns dahin stellen und dann wurde jede Einzelne reingeholt. Die Tür hinter sich zugemacht und dann war die Untersuchung dran. "Während der Untersuchungen waren nur Pflegerinnen und der behandelnde Arzt anwesend: „Nein, das haben die Schwestern gemacht. Die haben unten dann die Fußknöchel da mit solchen Manschetten da festgemacht, damit man sich nicht wehren konnte (...) Die Untersuchungen, das war der Horror für mich. Es war einfach ein Trauma für mich und da vergisst man alles andere auch dann ja, also nur die schlimmsten Sachen, die einen da wirklich passiert sind dann." ${ }^{467}$ Auch noch Mitte der 1980er Jahre fanden täglich die gynäkologischen Untersuchungen statt: „Na das ist eigentlich jeden Tag passiert und das war in einem Raum und wir mussten alle in der Schlange anstehen und es war auch offen, wer mit reingekommen ist. Und dann haben sie uns auch immer, wie soll ich sagen, so Wattebällchen mit irgendwas getränkt, verabreicht und haben behauptet, das ist ein Dings, was bewirken würde, dass die Krankheit ausbrechen würde. " ${ }^{468}$

Das pflegerische Personal war täglich mit den gynäkologischen Untersuchungen beschäftigt. Und obwohl die ungelernte Pflegerin beruflich nicht für die Tätigkeit auf der geschlossenen Venerologischen Station qualifiziert war, musste sie die Untersuchungen unterstützen: „Aber man wurde angelernt

466 Interview mit Frau LPG (Anm. 462).

467 Interview mit Frau LPF (Anm. 461).

468 Interview mit Frau LPG (Anm. 462). 
von den Schwestern und hat dann die Patienten versorgt. Also man war bei Untersuchungen zum Teil mit dabei. Man hat zugereicht oder auch mal Medikamente ausgegeben. (...) Also man wurde halt angelernt und dann so learning by doing. Also man hat halt mitbekommen, wie diese Erkrankungen sich so zeigen. Aber auch schon dadurch, dass es ja nicht so wie heute war, wo man ganz viel Literatur hatte, wo man das nachlesen konnte. Das war ja da nicht so. Also man musste sich auf das verlassen, was man so mündlich gesagt bekommen hat. (...) Also vormittags hatte man meistens die Untersuchungen. Also es wurden relativ häufig die Abstriche gemacht bei den Patienten. Also die zwei Haupterkrankungen, die dort behandelt wurden, das war in erster Linie die Gonorrhoe. Die Patienten, die blieben drei Wochen da. Also in einigen wenigen Fällen dann auch mal Lues oder so Chlamydien, halt solche Sachen, dass die dann da festgestellt wurden. (...) Die Abstriche, die wurden von den Schwestern gemacht. “ Für die gynäkologischen Untersuchungen wurde ein Clasbesteck verwendet. „Also so eine Art Clasröhre. Die wurde dann halt eingeführt. Das wurde mir dann auch gezeigt, wie man das macht und dann wurde mit so einem Abtupfer oder so, wurden die Abstriche dann gemacht. " 469 Die Glasröhren hatten einen Durchmesser von „zweieinhalb Zentimeter oder so. Also je nach dem, was dann halt gerade so vorhanden war. Also die wurden dann auch so sterilisiert. Also mit diesen Spritzen da, da gab es so ein Gerät dafür, soweit ich mich erinnere, auf der Station. Das wurde dann auf der Station selber sterilisiert wieder. " ${ }^{470} \mathrm{Zu}$ den Routineuntersuchungen gehörte Ende der 1980er Jahre auch ein HIV-Test. „Genau. Also, das weiß ich aus den Gesprächen mit den Schwestern. Also die Patienten haben alle einen HIV-Test bekommen. Aber in der Zeit, in der ich da war, also wurde keine Infektion festgestellt." 471

Eine Veränderung auf der geschlossenen Venerologischen Krankenstation Thonberg hatte sich Mitte der 1970er Jahre angekündigt und setzte sich in den 1980er Jahren durch: Die Mädchen und Frauen arbeiteten nach der gynäkologischen Untersuchung nicht mehr, sondern waren sich selbst überlassen. So schildert eine Zeitzeugin, die 1982 auf der Station war, den Alltag: Nach den Untersuchungen ,gab es Frühstück und den ganzen Tag passierte gar nichts, also wir waren auf uns selber gestellt, ja und, das heißt rumgammeln, rumstehen, warten auf den nächsten Tag. (...) Ja, nichts, gar nichts. Ich sagte ja, wir standen nur im Flur rum und einige Frauen, die haben da ihre Tütchen gedreht, da aus Kippen ja und, also rauchen durfte man da oben. Und ja, sonst waren wir auf uns selber gestellt den ganzen Tag. “472 Eine andere Zeitzeugin bestätigt diese Aussage. Wenn man wollte, dann „durfte man aber auch arbeiten gehen und zwar in dem Objekt, dann in der Wäscherei. Das war bissel

469 Interview mit Frau LSA (Anm. 464).

470 Interview mit Frau LSA (Anm. 464).

471 Interview mit Frau LSA (Anm. 464).

472 Interview mit Frau LPF (Anm. 461). 
nebenan (...), das war dann bissel auf, also man musste schon raus aus dem Objekt.“ An weitere Tätigkeiten konnte sich auch diese Zeitzeugin nicht erinnern: „Kann ich nicht mehr so sagen, aber wir sind ja gleich erkannt worden, weil wir ja alle die Einheitskleidung anhatten, wussten gleich, wo wir her sind." ${ }^{43}$ Auf der Station selbst waren die Mädchen und Frauen lediglich zum Bettenmachen und Putzen der Räume verpflichtet.

Auch die ehemalige Hilfspflegerin bestätigt, dass die Mädchen und Frauen kaum Beschäftigung hatten: „Die haben da im Aufenthaltsraum rumgesessen. (...) Die haben ja nur rumgesessen und geredet. Man hat auch selber mit den Patienten ein bisschen geredet ja. (...) Ich habe die auch gefragt oder so. Also da war jetzt auch nicht immer unbedingt die schlechte Stimmung. Also die Frauen haben da halt zusammengesessen, die haben auch mal ein Späßchen gemacht. Ja und dann während der Wendezeit und so war eh die Stimmung ein bisschen lockerer." Für Abwechslung sorgte manchmal die Essenausgabe: „Also es gab jemanden aus der Psychiatrie, der immer so das Essen rumgebracht hat oder so. Manchmal musste man das dann auch irgendwie holen, aber das wurde eigentlich von psychiatrischen Patienten gebracht. (...) Also es war eine geschlossene Station. Man hatte, also die war wirklich verschlossen und darauf hat man halt auch geachtet, dass da keiner ein- und ausgehen konnte. Deswegen war das die Ausnahme, dass die da mal rauskonnten. Es gab halt mal den Fall, dass dann mal zwei Patientinnen unter einem Vorwand abgehauen sind. (...) Die wurden dann gesucht. Da kann ich mich noch erinnern. Da war dann die Polizei da. Da musste man eine Patientenbeschreibung abgeben. Genau, die wurden dann polizeilich gesucht, aber ich weiß nicht, ob die dann wiedergefunden wurden." ${ }^{774}$

Nur die Mahlzeiten strukturierten den Tagesablauf. Sie wurden im Aufenthalts- bzw. Speiseraum eingenommen. Das Essen wurde geliefert: „[W]ir haben ja immer die Essenlieferung im Kübel gekriegt. Essenkübel, so und da durften auch zwei Frauen, also Patienten jetzt mit runter, weil die Kübel mussten ja hochgebracht, hochgetragen werden. Ja und da die Stationsschwester das nicht alleine schafften, haben sie immer mit Begleitung zwei Frauen mitgenommen. " Da der Aufenthalts- bzw. Speiseraum relativ klein war, konnte nur abwechselnd gegessen werden, ,weil der halt nicht so groß war, also so einen kleinen Raum, wo zwei, drei Tische drinnen standen“. Bei diesen Vorgängen, dem Essenholen, der Mahlzeit oder während der Freizeit, war stets medizinisch-pflegerisches Personal anwesend, die alles überwachten. „Wenn wir in dem Aufenthaltsraum waren, da immer eine von den Schwestern oder was, war immer jemand dabei." ${ }^{475} \mathrm{Um} 8 \mathrm{Uhr}$ war schließlich Bettruhe, zwei Stunden früher als in den 197oer Jahren.

473 Interview mit Frau LPG (Anm. 462).

474 Interview mit Frau LSA (Anm. 464).

475 Interview mit Frau LPF (Anm. 461). 
Eine Zeitzeugin erinnert sich, dass in den vier Wochen ihres Aufenthalts in Leipzig-Thonberg 30 Frauen zwangsinterniert waren. Diese 30 Frauen wurden durch eine Stubenälteste vertreten, wie es in der „Hausordnung für die Krankenstation Thonberg" von 1966 hieß, bzw. überwacht, wie es die Zeitzeuginnen beschreiben: „Da waren ja gerade mal 30 Mann dort, mehr waren ja nicht da. (...) Ja, erst einmal das und dann war auch eine Schwester da. Da war eine Schwester und die Stubenälteste. "Trotz der Überwachung durch das Pflegepersonal wurden die Jüngeren drangsaliert und unterdrückt: ,Ja, ich habe da Patienten kennengelernt, die teilweise auch eins, zwei Jahre jünger waren. Und da auch älter waren und die wussten mit sich selber auch nichts anzufangen dort und die Jüngeren, die mussten dann halt immer für die anderen, für die Älteren da halt immer alles machen. " Die Mädchen und jüngeren Frauen mussten „halt die Betten bauen und wenn man die nicht gebaut hat, dann hat man uns zusammengestaucht oder hat man uns wie ein Hund behandelt ja, musste man hinterherlaufen auf allen Vieren. (...) Als Spielzeug sozusagen. “ ${ }^{476}$ Auch Mitte der 1980er Jahre gab es eine Stubenälteste: „Man hatte eine Zimmerälteste gehabt. Da war es immer gut, wenn man mit der gut stand, weil dann durfte man halt unter der Woche öfters mal duschen, sonst durfte man bloß einmal in der Woche duschen. “477 An gewalttätige Übergriffe kann sich die ehemalige Zwangseingewiesene nicht erinnern - weder durch andere Zwangseingewiesene noch durch die Stubenältesten. Dennoch musste jedes besondere Vorkommen, das beobachtet wurde, vom pflegerischen Personal schriftlich vermerkt werden: „Es gab halt ein Buch in dem wurden handschriftlich so Vorkommnisse festgehalten, also die Patienten. Da stand dann halt irgendwie weglaufgefährdet oder das nannte sich damals, die sind geflitzt oder die wollten flitzen oder so oder haben das angekündigt, so hieß das da ja. Und dann wurde das da vermerkt und da sollte man irgendwie so reinschauen, ja. Und sich dann sozusagen einen Überblick über die Lage verschaffen, also wenn man da den Dienst übernommen hat. "In diesem Zusammenhang blieb der Zeitzeugin besonders der Isolierraum in Erinnerung: „Da gab es halt einen vergitterten, kleinen Raum. Da wurden halt Lues-Patienten isoliert. Die haben dann eigene Handtücher bekommen, eigene Utensilien, ja und die mussten dann da diese Zeit zubringen. (...) Also ja, ich weiß noch, dass zu meiner Zeit, wenn ich da war, dass das eigentlich leer stand. Da hatten sie keine. Also nur am Anfang, ich glaube, als ich angefangen habe, da war mal kurzzeitig eine Patientin da. Aber ansonsten war da eigentlich in der Regel niemand. Also es war trotzdem eine relativ, ja, seltene Erkrankung. Also ich erinnere mich an zwei Patientinnen. Also die eine Patientin, die mal Lues hatte, die im zweiten Stadium, also mit den Hautausschlägen und eine Patientin, die da mal isoliert war." ${ }^{478}$ 
Waren 1982 noch 30 Mädchen und Frauen auf der geschlossenen Venerologischen Krankenstation Thonberg zwangseingewiesen, reduzierte sich die Zahl 1985 auf 7 bis 8 Frauen auf der Station. Zu dieser Zeit fand auch ein Trägerwechsel der Einrichtung statt. Die geschlossene Venerologische Station gehörte nun zum Bezirkskrankenhaus für Psychiatrie Leipzig, Abteilung Venerologie. ${ }^{479}$ Seit dieser Zeit war im Gebäude der Riebeckstraße 63 im Erdgeschoss die „Psychiatrie. Wir waren im ersten Stock untergebracht und auch Gitter vor die Fenster und total eingesperrt. “ Die Mädchen und Frauen der geschlossenen Venerologischen Station waren von der Psychiatrie getrennt. „Man ist halt ziemlich abgeschottet worden, (...) dann ist man bloß in Aufenthaltsraum gekommen, wo bloß Stühle dort standen, keine Tische und nichts und konnte man sich da aufhalten und wenn es einem nicht gut ging, durfte man nicht mal ins Bett gehen, sondern konnte man sich da irgendwo auf die Stühle legen. “480 Und die ehemalige Hilfspflegekraft bestätigt: „[E]s war eine geschlossene Station, also, ja, das Untersuchungszimmer mit der Untersuchungsliege, daran erinnere ich mich. “ Die Fenster der Station waren vergittert: „Ja, also da waren so Gitter vor den Fenstern, ja. “481

Während eine Zeitzeugin Anfang der 1980er Jahre vier Wochen auf der Station zwangseingewiesen war, wurde eine andere Zeitzeugin Mitte der 1980er Jahre bereits nach 14 Tagen entlassen. „Ich weiß noch, wo meine Mutti mich abgeholt hat, die durfte klopfen, ich durfte nicht mal ,Guten Tag' sagen, weil ich habe sie vor der Tür stehen sehen." Während der Entlassung musste wie in den Jahren zuvor eine Schweigeerklärung von der damals 15-Jährigen unterschrieben werden. „Ja, das weiß ich, ja.“482

Über den Prozess der Auflösung der geschlossenen Venerologischen Station in Leipzig-Thonberg ist nur wenig bekannt. Wann die letzten Zwangseinweisungen stattfanden und wie die letzten Zwangseingewiesenen entlassen wurden, bleibt offen. Als die Hilfspflegerin im Frühjahr 1990 Leipzig verließ, war die Station noch in Betrieb. Sicher ist, dass die geschlossene Venerologische Station infolge der Wiedervereinigung aufgelöst wurde, denn die gesetzliche Grundlage, die „Verordnung zur Verhütung und Bekämpfung der Geschlechtskrankheiten“ vom 23. Februar 1961, wurde in dieser Zeit für die gesamte DDR aufgehoben. Damit war die rechtliche Grundlage für die Existenz der geschlossenen Venerologischen Stationen in der gesamten ehemaligen DDR aufgehoben worden. Heute befindet sich im Gebäude der Riebeckstraße 63 ein Heim für Asylbewerber. 